\title{
Global attractor for a non-autonomous integro-differential equation in materials with memory
}

\author{
T. Caraballo, M.J. Garrido-Atienza, \\ Dpto. de Ecuaciones Diferenciales y Análisis Numérico, Universidad de Sevilla, \\ Apdo. de Correos 1160, 41080 Sevilla, Spain \\ B. Schmalfuß
}

Institut für Mathematik, Fakultät EIM, Universität Paderborn, Warburger Strasse 100, 3309 Paderborn, (Germany)

$\&$

J. Valero

Centro de Investigación Operativa, Universidad Miguel Hernández, Avda. de la Universidad, s/n, 03202 Elche, (Spain)

\begin{abstract}
The long-time behavior of an integro-differential parabolic equation of diffusion type with memory terms, expressed by convolution integrals involving infinite delays and by a forcing term with bounded delay, is investigated in this paper. The assumptions imposed on the coefficients are weak in the sense that uniqueness of solutions of the corresponding initial value problems cannot be guaranteed. Then, it is proved that the model generates a multivalued non-autonomous dynamical system which possesses a pullback attractor. First, the analysis is carried out with an abstract parabolic equation. Then, the theory is applied to the particular integro-differential equation which is the objective of this paper. The general results obtained in the paper are also valid for other types of parabolic equations with memory.
\end{abstract}

Key words: Delayed reaction-diffusion equations, integro-differential equations with memory, non-autonomous (pullback) attractors, multivalued dynamical systems, asymptotic behavior.

Mathematics Subject Classifications (2000): 34K10, 35B40, 35B41, 35K55, 35K57, $35 \mathrm{Q} 35$ 


\section{Introduction}

The aim of this paper is to analyze the long-time behavior of solutions of an integro-differential parabolic equation of diffusion type with memory terms, expressed by convolution integrals involving infinite delays and by a forcing term with bounded delay, which represent the past history of one or more variables. In particular, we focus on the following non-autonomous reactiondiffusion equation with memory

$$
\frac{\partial u}{\partial t}-\Delta u+\int_{-\infty}^{t} \gamma(t-s) \Delta u(x, s) d s+g(x, t, u(x, t))=f_{1}(x, t, u(x, t-h)),
$$

with Dirichlet boundary condition, where $x$ belongs to a bounded domain $\mathcal{O} \subset \mathbb{R}^{N}$ with smooth boundary, $t \in \mathbb{R}$, the functions $f_{1}$ and $g$ satisfy suitable assumptions (see Section 4), and $\gamma$ is given in a standard way as $\gamma(t)=$ $-\gamma_{0} e^{-d_{0} t}$ with $d_{0}>$ and $\gamma_{0}>0$. For the definition and properties of the coefficients see below.

It is well known that many physical phenomena are better described if one considers in the equations of the model some terms which take into account the past history of the system. Although, in some situations, the contribution of the past history may not be so relevant to significantly affect the long time dynamics of the problem, in certain models, such as those describing high viscosity liquids at low temperatures, or the thermomechanical behavior of polymers (see [15], [28] and the references therein) the past history plays a nontrivial role.

On the other hand, it is sensible to assume that the models of certain phenomena from the real world are more realistic if some non-autonomous terms are also considered in the formulation. Moreover, even if we consider an autonomous model with certain kind of memory, unless the delay is constant, then the systems is better described by a non-autonomous differential equations (e.g. systems with variable delays, distributed delays, etc.).

The asymptotic behavior of a stochastic version of Eq. (1) (with an additive

ऋ Corresponding author: T. Caraballo

Partially supported by Ministerio de Ciencia e Innovación (Spain), FEDER (European Community) under grants MTM2008-00088, MTM2009-11820 and HA20050082, by Deutschen akademischen Austauschdienst ppp Austauchprogramm Az: 314/Al-e-dr, Consejería de Cultura y Educación (Comunidad Autónoma de Murcia) grant 00684/PI/04, and Consejería de Innovación, Ciencia y Empresa (Junta de Andalucía) grant P07-FQM-02468.

Email addresses: caraball@us.es (T. Caraballo), mgarrido@us.es (M.J.

Garrido-Atienza), schmalfuss@uni-paderborn.de (B. Schmalfuß),

jvalero@umh.es (J. Valero). 
noise) and with conditions ensuring uniqueness of the Cauchy problem was studied in [2].

In the papers [20], [21], [25], [26] it is considered a general system of reactiondiffusion equations (without delay) in which the nonlinear term satisfies dissipative and growth conditions which are not sufficient to ensure the uniqueness of the Cauchy problem. In this way, important applications as the complex Ginzburg-Landau equations can be also considered (see [20], [21] and also [31]). Using the theory of attractors for multivalued semiflows or processes, the asymptotic behavior of solutions is studied. For the same kind of systems, the existence of trajectory attractors is proved in [10], [11]. In [9], using also the method of trajectory attractors, the authors present a global scheme for the construction of connected trajectory and global attractors for heat equations with linear fading memory and with non-linear heat sources.

In [8], a linear integro-differential equation for a class of memory functions in a Hilbert space arising from heat conduction with memory is considered. In particular, sufficient and necessary conditions for stability and exponential stability in both finite and infinite dimensional cases are established. In [16], the authors are able to construct a Lyapunov functional associated with the dynamical system in an appropriate history phase space. The existence of global attractors for reaction-diffusion systems with finite delay and uniqueness of the Cauchy problem has been considered in [34]. Trajectory attractors for reaction-diffusion equations with an infinite-delay memory term and uniqueness of solutions have been proved to exist in [7].

We extend the results of these previous papers to equation (1) by considering a similar nonlinear term $g$ (as in [20]-[21]), not ensuring uniqueness of the Cauchy problem, when some delays are present. Also, as the terms appearing in the equation are non-autonomous, we construct a multivalued process associated to the problem and study the existence of pullback attractors for it.

From the technical point of view some new and challenging difficulties appear with respect to all these works. One memory term involves an infinite (unbounded) delay which is given by a convolution term and second order partial derivatives. The other one containing a bounded (finite) delay is a general continuous term satisfying very weak restrictions. Due to these facts, we study the existence of the global attractor in the space $\mathcal{H}$ given by measurable functions $t \mapsto u(t) \in H_{0}^{1}(\mathcal{O})$ with $\int_{-\infty}^{0} \int_{\mathcal{O}} e^{\lambda_{1} s}|\nabla u|^{2} d x d s<\infty$ such that their restriction on $[-h, 0]$ has a version in $C\left([-h, 0] ; L^{2}(\mathcal{O})\right)$, where $\lambda_{1}$ is the first eigenvalue of $-\Delta$ in $H_{0}^{1}(\mathcal{O})$. The main difficulty appears when we have to prove the asymptotic compactness of the multivalued process, as the usual methods of the energy inequality or the monotonicity method (used for example in [20], [21], [25]) do not seem to work for the convergence in the norm 
$\|\cdot\|_{L_{V}^{2}}$. Also, due to the absence of uniqueness it is also not possible to obtain suitable estimates in more regular spaces, as given in [2]. Nevertheless, as we will see later, the linearity of the infinite delayed term helps us to overcome these difficulties in other way.

Now we will describe how our model appears. The starting point for our considerations is the following heat conduction model.

Let $\mathcal{O}$ be a regular enough bounded domain in $\mathbb{R}^{N}$. We denote by $v=v(x, t)$ the temperature at position $x \in \overline{\mathcal{O}}$ and time $t$. Following the theory developed by Coleman \& Gurtin [13], Gurtin \& Pipkin [17] and Nunziato [27] we assume that the density $e(x, t)$ of the internal energy and the heat flux $q(x, t)$ are related to the temperature and its gradient by the constitutive relations:

$$
e(x, t)=b_{0} v(x, t), \quad t \in \mathbb{R}, x \in \overline{\mathcal{O}}
$$

and

$$
q(x, t)=-c_{0} \nabla v(x, t)+\int_{-\infty}^{t} \gamma(t-s) \nabla v(x, s) d s, \quad t \in \mathbb{R}, x \in \overline{\mathcal{O}} .
$$

Here the constants $b_{0}>0$ and $c_{0}>0$ are called respectively the heat capacity and the thermal conduction, $\gamma$ is the heat flux relaxation function (recall that the standard example is $\gamma(t)=-\gamma_{0} e^{-d_{0} t}$ with $d_{0}>0$ and $\left.\gamma_{0}>0\right)$.

The energy balance for the system has the form

$$
\partial_{t} e(x, t)=-\operatorname{div} q(x, t)+f(x, t, v(x, t)), \quad t \in \mathbb{R}, x \in \overline{\mathcal{O}},
$$

where $f(x, t, v)$ is the energy supply which may depend on the temperature. Thus we arrive at the following non-autonomous heat equation with memory

$$
b_{0} \partial_{t} v(x, t)=c_{0} \Delta v(x, t)-\int_{-\infty}^{t} \gamma(t-s) \Delta v(x, s) d s+f(x, t, v(x, t))
$$

where $t>0, x \in \mathcal{O}$. We also need to impose some (natural) boundary conditions for $v(x, t)$.

However, on some occasions it is sensible to think that the external forcing term $f$ may depend not only on the temperature at present time $t$ but also on some previous instant $t-h$ (for a positive $h>0$ ). This kind of situations come out very often in problems related to feedback control. Consequently, one may assume that, instead of the previous $f$, it could be better to consider

$$
f_{1}(x, t, v(x, t-h))-g(x, t, v(x, t)),
$$

which yields the initial formulation of our problem (1) (for $b_{0}=c_{0}=1$ ). 
The article is organized as follows. In Section 2 the definition of a multivalued non-autonomous dynamical system is stated. In particular, we introduce the concept of pullback attractor for this kind of non-autonomous dynamical systems. To follow our purpose to investigate the long-time behavior of the system (1) we proceed as follows. Instead of working directly with our problem, we first introduce in Section 3 an abstract non-autonomous PDE (which contains in particular our model) with coefficients satisfying weak conditions. These coefficients contain finite and infinite delay terms. In particular we do not assume Lipschitz continuity of all these coefficients. Then we show the existence of at least one weak solution for (1). The set of all weak solutions forms a multivalued non-autonomous dynamical system. The existence of pullback attractor is established in Section 4. Finally, in the last section we apply the general theory to our problem (1).

\section{Preliminaries}

We will recall the general theory of pullback attractors for multivalued nonautonomous dynamical systems as given in [5] (see also [3] for the theory of multivalued non-autonomous systems in terms of cocycles).

Let $X=\left(X, d_{X}\right)$ be a Polish space. Denote by $P(X)$ the sets of all non-empty subsets of $X$, and by $\mathbb{R}_{d}=\left\{(t, \tau) \in \mathbb{R}^{2}: t \geq \tau\right\}$.

We now introduce multivalued non-autonomous dynamical systems.

Definition 1 A multi-valued map $U: \mathbb{R}_{d} \times X \rightarrow P(X)$ is called a multivalued non-autonomous dynamical system (MNDS) or a process if the following properties hold

i) $U(\tau, \tau, \cdot)=\operatorname{id}_{X}$, for all $\tau \in \mathbb{R}$,

ii) $U(t, \tau, x) \subset U(t, s, U(s, \tau, x))$ for all $\tau \leq s \leq t, x \in X$.

It is called a strict MNDS if, moreover, $U(t, \tau, x)=U(t, s, U(s, \tau, x))$ for all $\tau \leq s \leq t, x \in X$.

In order to define the concept of attractor we need to recall some other definitions.

Let $D: \mathbb{R} \rightarrow P(X)$ denote a multivalued mapping. $D$ is said to be negatively (resp. strictly) invariant for the MNDS $U$ if $D(t) \subset U(t, \tau, D(\tau))$ (resp. $=$ ), for $(t, \tau) \in \mathbb{R}_{d}$.

Let $\mathcal{D}$ be a family (or universe) of multivalued mappings $(D(\tau))_{\tau \in \mathbb{R}}$. We say 
that a family $K$ is pullback $\mathcal{D}$-attracting if for every $D \in \mathcal{D}$

$$
\lim _{\tau \rightarrow+\infty} \operatorname{dist}_{X}(U(t, t-\tau, D(t-\tau)), K(t))=0, \text { for all } t \in \mathbb{R}
$$

where by $\operatorname{dist}_{X}(A, B)$ we denote the Hausdorff semi-distance of two non-empty sets $A, B: \operatorname{dist}_{X}(A, B)=\sup _{x \in A} \inf _{y \in B} d_{X}(x, y)$.

$B$ is said to be pullback $\mathcal{D}$-absorbing if for every $D \in \mathcal{D}$ and $t \in \mathbb{R}$ there exists $T=T(t, D)>0$ such that

$$
U(t, t-\tau, D(t-\tau)) \subset B(t), \text { for all } \tau \geq T \text {. }
$$

Throughout this work we always consider a particular system of sets as in [30]. Namely, let $\mathcal{D}$ be a set of multivalued mappings $D: \tau \mapsto D(\tau) \in P(X)$ (i.e. with non-empty images) satisfying the inclusion closed property: suppose that $D \in \mathcal{D}$ and let $D^{\prime}$ be a multivalued mapping $D^{\prime}: \tau \mapsto D^{\prime}(\tau) \in P(X)$ such that $D^{\prime}(\tau) \subset D(\tau)$ for $\tau \in \mathbb{R}$, then $D^{\prime} \in \mathcal{D}$. It is remarkable that in considering such a system of sets, we will be able to prove the uniqueness of the pullback attractor in $\mathcal{D}$.

For some element $B \in \mathcal{D}$, an MNDS is said to be $\mathcal{D}$-asymptotically compact with respect to $B$ if for every sequence $\tau_{n} \rightarrow+\infty$ and $t \in \mathbb{R}$, it holds that every sequence $y_{n} \in U\left(t, t-\tau_{n}, B\left(t-\tau_{n}\right)\right)$ is pre-compact.

Let us define a global pullback $\mathcal{D}$-attractor.

Definition 2 A family $\mathcal{A} \in \mathcal{D}$ is said to be a global pullback $\mathcal{D}$-attractor for the MNDS $U$ if it satisfies:

i) $\mathcal{A}(t)$ is compact for any $t \in \mathbb{R}$;

ii) $\mathcal{A}$ is pullback $\mathcal{D}$-attracting;

iii) $\mathcal{A}$ is negatively invariant.

$\mathcal{A}$ is said to be a strict global pullback $\mathcal{D}$-attractor if the invariance property in the third item is strict.

Now we can formulate the following theorem, proved in [5] (see also [3] for a more general non-autonomous and random framework).

Theorem 3 Suppose that the $M N D S U(t, \tau, \cdot)$ is upper-semicontinuous for $(t, \tau) \in \mathbb{R}_{d}$ and possesses closed values. Let $B \in \mathcal{D}$ be a multivalued mapping such that the $M N D S$ is $\mathcal{D}$-asymptotically compact with respect to $B$. In addition, suppose that $B$ is pullback $\mathcal{D}$-absorbing. Then, the set $\mathcal{A}$ given by

$$
\mathcal{A}(t):=\bigcap_{\tau \geq 0} \overline{\bigcup_{s \geq \tau} U(t, t-s, B(t-s))}
$$


is a pullback $\mathcal{D}$-attractor. Furthermore, $\mathcal{A}$ is the unique element from $\mathcal{D}$ with these properties. In addition, if $U$ is a strict $M N D S$, then $\mathcal{A}$ is strictly invariant.

\section{Existence of solutions of the integro-differential equation}

We intend now to introduce a setting to find a solution of the problem (1). However, instead of working directly with our model, we will consider an abstract problem (which contains our problem as a particular case) and with a little additional work, we will cover other equations at the same time.

Let $\mathcal{O}$ be a bounded domain in $\mathbb{R}^{N}$ with smooth boundary. On this set we introduce the space $L^{p}(\mathcal{O})$ with norm $|\cdot|_{p}$ for $p>1$. We denote by $\langle\cdot, \cdot\rangle_{q}$ the pairing between $L^{p}(\mathcal{O})$ and $L^{q}(\mathcal{O}), \frac{1}{q}+\frac{1}{p}=1$. The space $L^{2}(\mathcal{O})$ is also denoted by $H$ and its norm and scalar product are denoted by $\|\cdot\|,(\cdot, \cdot)$. We also have the Sobolev spaces $W_{2}^{s}(\mathcal{O})=H^{s}(\mathcal{O})$ of functions with generalized derivatives up to the order $s \in \mathbb{N}$ in $L^{2}(\mathcal{O})$ (see [24] for the definition in the case where $s$ is not an integer). Let $H_{0}^{s}(\mathcal{O})$ be the closure of $C_{0}^{\infty}(\mathcal{O})$ with respect to these norms in $H^{s}(\mathcal{O})$ and denote $V=H_{0}^{1}(\mathcal{O})$.

We now consider uniformly elliptic differential operators of second order

$$
A(x, D)=-\sum_{i, j=1}^{N} D_{i}\left(a_{i j}(x)\right) D_{j}
$$

with homogenous Dirichlet boundary conditions $\left.u\right|_{\partial \mathcal{O}}=0$ defined on sufficiently smooth functions. In particular, we suppose that $a_{i j}=a_{j i} \in C^{\infty}(\overline{\mathcal{O}})$. Then we know that we can extend the above differential operator to a positive operator $A$ defined on $H_{0}^{1}(\mathcal{O}) \cap H^{2}(\mathcal{O})$. This operator has a compact inverse with respect $H$. Hence this operator has a discrete positive spectrum $0<\lambda_{1} \leq \lambda_{2} \leq \cdots$ of finite multiplicity and

$$
\lim _{n \rightarrow \infty} \lambda_{n}=\infty .
$$

with associated eigenelements of $A$ denoted by $e_{1}, e_{2}, \cdots$ generating a complete orthonormal system in $H$.

We also define the spaces

$$
V^{\alpha}=\left\{u \in \mathcal{D}^{\prime}(\mathcal{O}): u=\sum_{i=1}^{\infty} \hat{u}_{i} e_{i}, \hat{u}_{i}=\left\langle u, e_{i}\right\rangle_{\mathcal{D}^{\prime}(\mathcal{O})},\|u\|_{\alpha}^{2}=\sum_{i=1}^{\infty} \lambda_{i}^{\alpha}\left|\hat{u}_{i}\right|^{2}<\infty\right\}
$$

where, as usual, $\mathcal{D}^{\prime}(\mathcal{O})$ denotes the distributions space over $\mathcal{O}$. We have $V^{0}=$ $H, V^{1}=V, V^{-1}=V^{\prime}$. The duality between $V^{\alpha}$ and $V^{-\alpha}=\left(V^{\alpha}\right)^{\prime}$ is denoted 
by $\langle\cdot, \cdot\rangle$. By a bootstrap argument it follows that $e_{i} \in V^{\alpha}$ for $\alpha \in \mathbb{R}$. In particular, we have

$$
\langle A u, u\rangle=\|u\|_{1}^{2} \quad \text { for } u \in V
$$

The following embedding theorem is well known (see [32, Lemma 2.1 in Chapter 4]):

Lemma 4 (i) Suppose that $p \geq 2$, and

$$
s \geq N\left(\frac{1}{2}-\frac{1}{p}\right)
$$

Then we have the continuous embedding $H^{s}(\mathcal{O}) \subset L^{p}(\mathcal{O})$.

(ii) Suppose that $\alpha \geq s$ for $s \in \mathbb{N}$. Then we have the continuous embedding

$$
V^{\alpha} \subset H^{s}(\mathcal{O}) \text {. }
$$

As a consequence or Lemma 4 (see also [29, Section 8.2]) it follows that $e_{i} \in$ $V^{s} \subset H^{s}(\mathcal{O}) \subset L^{p}(\mathcal{O})$, for $s \geq N\left(\frac{1}{2}-\frac{1}{p}\right)$, and $\left\{e_{j}\right\}_{j=1}^{\infty}$ is complete in $H_{0}^{1}(\mathcal{O}) \cap$ $L^{p}(\mathcal{O})$.

Let $C([a, b] ; H)$ be the space of continuous functions $u:[a, b] \mapsto H, a<b \in \mathbb{R}$ equipped with the standard supremum norm. In particular, we consider this space often for $a=-h$ and $b=0$ which is then denoted by $C_{h}$ with norm $\|\cdot\|_{C_{h}}$. By $L^{2}\left(a, b ; V^{\alpha}\right),-\infty<a<b<\infty$ we denote the space of measurable mappings $u:(a, b) \mapsto u(t) \in V^{\alpha}$ such that

$$
\|u\|_{L^{2}\left(a, b ; V^{\alpha}\right)}^{2}:=\int_{a}^{b}\|u(\tau)\|_{\alpha}^{2} d \tau<\infty .
$$

A mapping $\psi(t) \in V$ for a.e. $t \in(-\infty, T)$ is an element of the space $L^{2}(-\infty, T ; V)$ if

$$
\|\psi\|_{L^{2}(-\infty, T ; V)}^{2}=\int_{-\infty}^{T} e^{\lambda_{1} s}\|\psi(s)\|_{1}^{2} d s<\infty .
$$

We also use the abbreviation $L_{V}^{2}=L^{2}(-\infty, 0 ; V)$.

For a function $u \in L^{2}(-\infty, t ; V)$ we will write $u_{t}=u(t+\cdot) \in L_{V}^{2}$ for $t \in \mathbb{R}^{+}$. The following space is the state space investigating the dynamics of (1). Let $h$ be a positive constant and $p \geq 2$. We set $\mathcal{H}$ to be the space of functions in $L_{V}^{2}$ such that their restriction on $[-h, 0]$ has a version in $C_{h}$. This space is equipped with the norm

$$
\|u\|_{\mathcal{H}}^{2}=\|u\|_{L_{V}^{2}}^{2}+\|u\|_{C_{h}}^{2} .
$$

It is straightforward that this space is a separable Banach space. 
We aim to analyze the following non-autonomous evolution equation

$$
\frac{d u}{d t}+A u=F_{2}\left(t, u_{t}\right)+F_{1}\left(t, u_{t}\right)-G(t, u), \quad u(\tau+s)=\psi(s) \quad \text { for } s \leq 0,
$$

where $\tau \in \mathbb{R}$, the operator $A$ has been introduced at the beginning of this section, $\psi \in \mathcal{H}$ and

$$
\begin{aligned}
& G: \mathbb{R} \times L^{p}(\mathcal{O}) \rightarrow L^{q}(\mathcal{O}), \\
& F_{1}: \mathbb{R} \times C_{h} \rightarrow H \\
& F_{2}: \mathbb{R} \times L_{V}^{2} \rightarrow V^{\prime}
\end{aligned}
$$

are continuous operators satisfying the following assumptions: for some positive constants $\eta, \rho$ and positive functions $c_{1}, c_{2} \in L_{l o c}^{1}(\mathbb{R})$, it holds

$$
\begin{aligned}
& \langle G(t, v), v\rangle_{q} \geq \eta|v|_{p}^{p}-c_{1}(t), \\
& |G(t, v)|_{q}^{q} \leq \rho|v|_{p}^{p}+c_{2}(t), \text { for } v \in L^{p}(\mathcal{O}) .
\end{aligned}
$$

We also assume

$$
\left\|F_{1}(t, \xi)\right\| \leq c_{3}(t)+c_{4}(t)\|\xi\|_{C_{h}}, \text { for } \xi \in C_{h}
$$

where $c_{3}, c_{4}$ are positive functions such that $c_{3}^{2}, c_{4}^{2} \in L_{\text {loc }}^{1}(\mathbb{R})$. On the other hand, we assume that there is a $d \in(0,1)$ and a positive function $c_{5} \in L_{l o c}^{1}(\mathbb{R})$ such that

$$
2 \int_{\tau}^{t} e^{\lambda_{1} s}\left\|F_{2}\left(s, u_{s}\right)\right\|_{-1}^{2} d s \leq \int_{\tau}^{t} e^{\lambda_{1} s} c_{5}(s) d s+\frac{d}{2} \int_{-\infty}^{t} e^{\lambda_{1} s}\|u(s)\|_{1}^{2} d s,
$$

for all $\tau \in \mathbb{R}, t \geq \tau$ and $u \in L^{2}(-\infty, t ; V)$. In addition, there exist a $K>0$ and a positive function $c_{6} \in L_{l o c}^{1}(\mathbb{R})$ for which

$$
2\left\|F_{2}(t, \psi)\right\|_{-1}^{2} \leq c_{6}(t)+K\|\psi\|_{L_{V}^{2}}^{2} \text {, for } \psi \in L_{V}^{2}, \text { and for } t \in \mathbb{R} .
$$

Assume that for a sequence $\left(u^{n}\right)_{n \in \mathbb{N}}$ the convergences $u^{n} \rightarrow u$ in $L^{2}(\tau, T ; H)$, $u^{n} \rightarrow u$ weakly in $L^{p}\left(\tau, T ; L^{p}(\mathcal{O})\right)$ and $u^{n} \rightarrow u$ weakly in $L^{2}(-\infty, T ; V)$ imply that

$$
\begin{gathered}
G\left(\cdot, u^{n}(\cdot)\right) \rightarrow G(\cdot, u(\cdot)) \text { weakly in } L^{q}\left(\tau, T ; L^{q}(\mathcal{O})\right) \\
F_{2}\left(\cdot, u_{.}^{n}\right) \rightarrow F_{2}(\cdot, u .) \text { weakly in } L^{2}\left(\tau, T ; V^{\prime}\right),
\end{gathered}
$$

and

$$
\begin{gathered}
\lim \inf _{n \rightarrow \infty} \int_{\tau}^{T} e^{-\lambda_{1}(T-s)}\left\langle G\left(s, u^{n}(s)\right), u^{n}(s)\right\rangle_{q} d s \\
\geq \int_{\tau}^{T} e^{-\lambda_{1}(T-s)}\langle G(s, u(s)), u(s)\rangle_{q} d s,
\end{gathered}
$$

for every $\tau \in \mathbb{R}$ and $T>\tau$. 
We also need the following assumption: for all $t>\tau, \tau \in \mathbb{R}, u, v \in L^{2}(-\infty, t ; V)$ we have

$$
2 \int_{\tau}^{t} e^{\lambda_{1} s}\left\|F_{2}\left(s, u_{s}\right)-F_{2}\left(s, v_{s}\right)\right\|_{-1}^{2} d s \leq \frac{b}{2} \int_{-\infty}^{t} e^{\lambda_{1} s}\|u(s)-v(s)\|_{1}^{2} d s,
$$

where $0<b<1$.

Definition 5 A function $u$ defined on $\mathbb{R}$ is said to be a weak solution, with initial function $\psi \in \mathcal{H}$, to the non-autonomous evolution equation (3) if for every $t \geq \tau$ we have that $u_{t} \in \mathcal{H}$, the restriction of $u$ on any interval $[\tau, T]$ is in $L^{p}\left(\tau, T ; L^{p}(\mathcal{O})\right)$, u has a derivative $\partial_{t} u$ in $L^{2}\left(\tau, T ; V^{\prime}\right)+L^{q}\left(\tau, T ; L^{q}(\mathcal{O})\right)$, so that

$$
u(t)-u\left(t_{0}\right)=\int_{t_{0}}^{t} \partial_{t} u(s) d s \quad \text { holds for } \tau \leq t_{0} \leq t \leq T,
$$

and $u$ satisfies the equation for every $t \geq \tau$, i.e.

$$
u(t)-u(\tau)+\int_{\tau}^{t} A u(s) d s=\int_{\tau}^{t}\left(F_{2}\left(s, u_{s}\right)+F_{1}\left(s, u_{s}\right)-G(s, u(s))\right) d s,
$$

where the equality is understood in the sense of $V^{\prime}+L^{q}(\mathcal{O})$. In other words, for any $e_{j}, j \geq 1$, it holds

$\left(u(t), e_{j}\right)=\left(u(\tau), e_{j}\right)+\int_{\tau}^{t}\left(-A u(s)+\left(F_{2}\left(s, u_{s}\right)+F_{1}\left(s, u_{s}\right)-G(s, u(s)), e_{j}\right) d s\right.$.

Notice that $\left\{e_{j}\right\}_{j \geq 1}$ is a dense set in $V \cap L^{p}(\mathcal{O})$.

Since $\frac{d\|u(t)\|^{2}}{d t}=2\left\langle\partial_{t} u(t), u(t)\right\rangle_{Y}$ a.e. $t \in[\tau, T]$, where $\langle\cdot, \cdot\rangle_{Y}$ denotes pairing between $Y=V^{\prime}+L^{q}(\mathcal{O})$ and $V \cap L^{p}(\mathcal{O})$ (see [10], [11]), it holds the energy equality

$\frac{d\|u(t)\|^{2}}{d t}+2\|u(t)\|_{1}^{2}=2\left\langle F_{2}\left(t, u_{t}\right), u(t)\right\rangle+2\left(F_{1}\left(t, u_{t}\right), u(t)\right)-2\langle G(t, u(t)), u(t)\rangle_{q}$,

for a.a. $t \in[\tau, T]$. Also, the function $u:[\tau, T] \rightarrow H$ is continuous.

We will use the notation $u(\cdot ; \tau, \psi)$ to denote a weak solution of $(3)$, but we will simply write $u(\cdot)$ when no confusion is possible.

We now formulate the main theorem of this section.

Theorem 6 Assume conditions (4)-(10). Then, for every initial function $\psi \in$ $\mathcal{H}$ there exists at least one weak solution $u$ to equation (3). In particular, we have $u_{t} \in \mathcal{H}$ for every $t \geq \tau$ and the restriction of $u$ on $[\tau, T]$ is contained $u \in L^{p}\left(\tau, T ; L^{p}(\mathcal{O})\right)$ for $T>\tau$. 
The proof of this theorem is divided into several lemmata.

Let $P_{n}: V^{\alpha} \rightarrow V^{\alpha}, \alpha \in \mathbb{R}$, be the orthogonal projection onto the space spanned by the first $n$ eigenelements of the basis introduced above. The associated linear space is denoted by $V_{n}$. We consider the Galerkin approximations to $(3)$.

For every fixed $n$ we define

$$
u^{n}(t)=\sum_{j=1}^{n} \gamma_{j}^{n}(t) e_{j}
$$

where the coefficients $\gamma_{j}^{n}$ are required to satisfy the following system:

$$
\begin{aligned}
\frac{d}{d t}\left(u^{n}(t), e_{j}\right)+\left(A u^{n}(t), e_{j}\right) & =\left(F_{2}\left(t, u_{t}^{n}\right)+F_{1}\left(t, u_{t}^{n}\right)-G\left(t, u^{n}(t)\right), e_{j}\right), \\
\psi^{n}(s+\tau) & =P_{n} \psi(s), \text { for } s \leq \tau,
\end{aligned}
$$

for $1 \leq j \leq n$. Following [18, Theorem 1.1, page 36] the properties on $P_{n} F_{2}, P_{n} G, P_{n} F_{1}, P_{n} A$ ensure the following:

Lemma 7 There exists at least one local solution to (15) in the space $\mathcal{H}_{n}=$ $L^{2}\left(-\infty,-h ; V_{n}\right) \times C\left([-h, 0], V_{n}\right)$.

To conclude that these solutions are global we need some a priori estimates for these solutions with respect to the interval of existence. However we only present here a method to prove that if solutions for the original problem (3) exist, then these solutions satisfy special a priori estimates. This method can be also used for the Galerkin approximations to see that any solution of (15) is global.

Note at first that, by Young's inequality for $p>2$ and for every $\mu>0$, there exists $C_{\mu}>0$ such that

$$
|u|_{p}^{p} \geq \mu\|u\|^{2}-C_{\mu}, \text { for } u \in L^{p}(\mathcal{O})
$$

When $p=2$ the same estimate is true with $\mu=1, C_{\mu}=0$.

Lemma 8 Under conditions (5)-(7), every weak solution u of (3) satisfies the estimates:

$$
\begin{aligned}
\left\|u_{t}\right\|_{C_{h}}^{2} \leq & 2 e^{-\lambda_{1}(t-\tau-h)+\int_{\tau}^{t} \frac{4 e^{\lambda_{1} h}}{\eta \mu} c_{4}^{2}(s) d s}\|\psi\|_{\mathcal{H}}^{2} \\
& +2 e^{\lambda_{1} h} \int_{\tau-t}^{0} e^{\lambda_{1} s+\int_{s}^{0} \frac{4 e^{\lambda_{1} h}}{\eta \mu} c_{4}^{2}(t+r) d r} c(s+t) d s
\end{aligned}
$$


and

$$
\begin{aligned}
\left\|u_{t}\right\|_{L_{V}^{2}}^{2} \leq & k e^{-\lambda_{1}(t-\tau-h)+\int_{\tau}^{t} \frac{4 e^{\lambda_{1} h}}{\eta \mu} c_{4}^{2}(s) d s}\|\psi\|_{\mathcal{H}}^{2} \\
& +\frac{4 e^{\lambda_{1} h}}{1-d} \int_{\tau-t}^{0} e^{\lambda_{1} s+\int_{s}^{0} \frac{4 e^{\lambda_{1} h}}{\mu \eta} c_{4}^{2}(r+t) d r} c(s+t) d s,
\end{aligned}
$$

for all $t \geq \tau$, where $k>0, c(t)=\eta C_{\mu}+c_{5}(t)+2 c_{1}(t)+\frac{2 c_{3}^{2}(t)}{\eta \mu}$, and $\mu, C_{\mu}$ are defined by (16).

Proof. Using (14), (2), (5) and (6) we derive, for $t \geq \tau$, the following energy inequality

$$
\begin{aligned}
\frac{d\|u\|^{2}}{d t}+\lambda_{1}\|u\|^{2}+\|u\|_{1}^{2}+2 \eta|u|_{p}^{p} \leq & 2\left\|F_{2}\left(t, u_{t}\right)\right\|_{-1}^{2}+\frac{1}{2}\|u\|_{1}^{2}+2 c_{1}(t) \\
& +2\left(c_{3}(t)+c_{4}(t)\left\|u_{t}\right\|_{C_{h}}\right)\|u\| .
\end{aligned}
$$

Hence, from (16), for $t \geq \tau$,

$$
\begin{aligned}
\frac{d\|u\|^{2}}{d t}+\lambda_{1}\|u\|^{2}+\|u\|_{1}^{2}+\eta|u|_{p}^{p} \leq & 2\left\|F_{2}\left(t, u_{t}\right)\right\|_{-1}^{2}+\frac{1}{2}\|u\|_{1}^{2}+2 c_{1}(t) \\
& +\eta C_{\mu}+\frac{2 c_{3}^{2}(t)}{\eta \mu}+\frac{2 c_{4}^{2}(t)}{\eta \mu}\left\|u_{t}\right\|_{C_{h}}^{2} .
\end{aligned}
$$

Then, for $C_{\eta, \mu}=\eta C_{\mu}$ and $t \geq \tau$, Gronwall's lemma yields

$$
\begin{aligned}
\| u(t) & \left\|^{2}+\frac{1}{2} \int_{\tau}^{t} e^{-\lambda_{1}(t-s)}\right\| u(s) \|_{1}^{2} d s \\
\leq & e^{-\lambda_{1}(t-\tau)}\|\psi(0)\|^{2}+2 \int_{\tau}^{t} e^{-\lambda_{1}(t-s)}\left\|F_{2}\left(s, u_{s}\right)\right\|_{-1}^{2} d s \\
& \quad+\int_{\tau}^{t} e^{-\lambda_{1}(t-s)}\left(C_{\eta, \mu}+2 c_{1}(s)+\frac{2 c_{3}^{2}(s)}{\mu \eta}+\frac{2 c_{4}^{2}(s)}{\mu \eta}\left\|u_{s}\right\|_{C_{h}}^{2}\right) d s .
\end{aligned}
$$

By (7) we have

$$
\begin{aligned}
& 2 \int_{\tau}^{t} e^{-\lambda_{1}(t-s)}\left\|F_{2}\left(s, u_{s}\right)\right\|_{-1}^{2} d s \\
& \quad \leq \int_{\tau}^{t} e^{-\lambda_{1}(t-s)} c_{5}(s) d s+\frac{d}{2} \int_{-\infty}^{\tau} e^{-\lambda_{1}(t-s)}\|u(s)\|_{1}^{2} d s+\frac{d}{2} \int_{\tau}^{t} e^{-\lambda_{1}(t-s)}\|u(s)\|_{1}^{2} d s \\
& \quad \leq \int_{\tau}^{t} e^{-\lambda_{1}(t-s)} c_{5}(s) d s+\frac{d}{2} e^{-\lambda_{1}(t-\tau)}\|\psi\|_{L_{V}^{2}}^{2}+\frac{d}{2} \int_{\tau}^{t} e^{-\lambda_{1}(t-s)}\|u(s)\|_{1}^{2} d s,
\end{aligned}
$$


and thus

$$
\begin{aligned}
& \|u(t)\|^{2}+\frac{1-d}{2} \int_{\tau}^{t} e^{-\lambda_{1}(t-s)}\|u(s)\|_{1}^{2} d s \\
& \quad=\|u(t)\|^{2}+\frac{1-d}{2} \int_{\tau-t}^{0} e^{\lambda_{1} s}\left\|u_{t}(s)\right\|_{1}^{2} d s \\
& \quad \leq e^{-\lambda_{1}(t-\tau)}\left(\|\psi(0)\|^{2}+\frac{d}{2}\|\psi\|_{L_{V}^{2}}^{2}\right)+\int_{\tau}^{t} e^{-\lambda_{1}(t-s)}\left(c(s)+\frac{2 c_{4}^{2}(s)}{\eta \mu}\left\|u_{s}\right\|_{C_{h}}^{2}\right) d s
\end{aligned}
$$

for $t \geq \tau$, where $c(t)=C_{\mu, \eta}+c_{5}(t)+2 c_{1}(t)+\frac{2 c_{3}^{2}(t)}{\eta \mu}$. Then

$$
\begin{aligned}
\left\|u_{t}\right\|_{C_{h}}^{2} \leq & e^{-\lambda_{1}(t-\tau-h)}\left(\|\psi(0)\|^{2}+\frac{d}{2}\|\psi\|_{L_{V}^{2}}^{2}\right) \\
& +e^{\lambda_{1} h} \int_{\tau}^{t} e^{-\lambda_{1}(t-s)}\left(c(s)+\frac{2 c_{4}^{2}(s)}{\eta \mu}\left\|u_{s}\right\|_{C_{h}}^{2}\right) d s
\end{aligned}
$$

for $t \geq \tau+h$. We note that if $\tau \leq t<\tau+h$, then we can obtain the same estimate for $\sup _{\theta \in[-(t-\tau), 0]}\|u(t+\theta)\|^{2}$ and for $\sup _{\theta \in[-h,-(t-\tau)]}\|u(t+\theta)\|^{2}=$ $\sup _{s \in[\tau-h, \tau]}\|u(s)\|^{2} \leq\|\psi\|_{C_{h}}^{2} \leq e^{-\lambda_{1}(t-\tau-h)}\|\psi\|_{C_{h}}^{2}$. Then

$$
\begin{aligned}
\left\|u_{t}\right\|_{C_{h}}^{2} \leq & e^{-\lambda_{1}(t-\tau-h)}\left(\|\psi\|_{C_{h}}^{2}+\frac{d}{2}\|\psi\|_{L_{V}^{2}}^{2}\right) \\
& +e^{\lambda_{1} h} \int_{\tau}^{t} e^{-\lambda_{1}(t-s)}\left(c(s)+\frac{2 c_{4}^{2}(s)}{\eta \mu}\left\|u_{s}\right\|_{C_{h}}^{2}\right) d s
\end{aligned}
$$

for all $t \geq \tau$, and we can conclude that

$$
\begin{aligned}
& \left\|u_{t}\right\|_{C_{h}}^{2}+\frac{1-d}{2} \int_{\tau-t}^{0} e^{\lambda_{1} r}\left\|u_{t}(r)\right\|_{1}^{2} d r \\
& \leq e^{-\lambda_{1}(t-\tau-h)}\left(2\|\psi\|_{C_{h}}^{2}+d\|\psi\|_{L_{V}^{2}}^{2}\right) \\
& +e^{\lambda_{1} h} \int_{\tau}^{t} e^{-\lambda_{1}(t-s)}\left(2 c(s)+4 \frac{c_{4}^{2}(s)}{\eta \mu}\left(\left\|u_{s}\right\|_{C_{h}}^{2}+\frac{1-d}{2} \int_{\tau-s}^{0} e^{\lambda_{1} r}\left\|u_{s}(r)\right\|_{1}^{2} d r\right)\right) d s .
\end{aligned}
$$

The Gronwall lemma implies for any $t \geq \tau$,

$$
\begin{aligned}
\left\|u_{t}\right\|_{C_{h}}^{2}+ & \frac{1-d}{2} \int_{\tau-t}^{0} e^{\lambda_{1} r}\left\|u_{t}(r)\right\|_{1}^{2} d r \\
\leq & e^{-\lambda_{1}(t-\tau-h)+\int_{\tau}^{t} \frac{4 e^{\lambda_{1} h}}{\eta \mu} c_{4}^{2}(s) d s}\left(2\|\psi\|_{C_{h}}^{2}+d\|\psi(s)\|_{L_{V}^{2}}^{2}\right) \\
& +2 e^{\lambda_{1} h} \int_{\tau}^{t} e^{-\lambda_{1}(t-r)+\int_{r}^{t} \frac{4 e^{\lambda_{1} h}}{\mu \eta} c_{4}^{2}(s) d s} c(r) d r \\
\leq & 2 e^{-\lambda_{1}(t-\tau-h)+\int_{\tau}^{t} \frac{4 e^{\lambda_{1} h}}{\eta \mu} c_{4}^{2}(s) d s}\|\psi\|_{\mathcal{H}}^{2} \\
& +2 e^{\lambda_{1} h} \int_{\tau-t}^{0} e^{\lambda_{1} s+\int_{s}^{0} \frac{4 e^{\lambda_{1} h}}{\eta \mu} c_{4}^{2}(t+r) d r} c(s+t) d s
\end{aligned}
$$

We have therefore proved (17). 
On the other hand, as a direct consequence of (17), for any $t \geq \tau$,

$$
\begin{aligned}
\frac{1-d}{2}\left\|u_{t}\right\|_{L_{V}^{2}}^{2}= & \frac{1-d}{2} \int_{-\infty}^{\tau-t} e^{\lambda_{1} s}\|\psi(t+s-\tau)\|_{1}^{2} d s+\frac{1-d}{2} \int_{\tau-t}^{0} e^{\lambda_{1} s}\left\|u_{t}(s)\right\|_{1}^{2} d s \\
\leq & \frac{1-d}{2} e^{-\lambda_{1}(t-\tau)}\|\psi\|_{L_{V}^{2}}^{2}+2 e^{-\lambda_{1}(t-\tau-h)+\int_{\tau}^{t} \frac{t e^{\lambda_{1}}}{\eta \mu} c_{4}^{2}(s) d s}\|\psi\|_{\mathcal{H}}^{2} \\
& +2 e^{\lambda_{1} h} \int_{\tau-t}^{0} e^{\lambda_{1} s+\int_{s}^{0} \frac{4 e^{\lambda_{1} h}}{\eta \mu} c_{4}^{2}(t+r) d r} c(s+t) d s
\end{aligned}
$$

and then (18) is also proved.

Throughout all the next results, $C$ denotes a generic positive constant, whose value is not so important and that may change from line to line. We write $C(\cdot)$ if the dependence of some parameters is crucial.

Corollary 9 Under conditions (5)-(8), for every bounded set $B$ in $\mathcal{H}$ and for any $T>\tau$, there exists a positive constant $C=C(T, B)$ such that for every weak solution $u(\cdot ; \tau, \psi)$ of (3) corresponding to the initial data $\psi \in B$ we have

$$
\|u(\cdot ; \tau, \psi)\|_{L^{p}\left(\tau, T ; L^{p}(\mathcal{O})\right)} \leq C, \quad \forall \psi \in B
$$

Proof. By Lemma 8 every weak solution $u$ is bounded in $L^{2}(-\infty, T ; V)$, with $\left\|u_{t}\right\|_{C_{h}}$ uniformly bounded, for any $T>\tau$ and $t \in[\tau, T]$. Further, by (8) we obtain that $F_{2}(\cdot, u$. $)$ is bounded in $L^{2}\left(\tau, T ; V^{\prime}\right)$. The estimate therefore follows by integrating $(20)$.

Lemma 10 Under conditions (5)-(8), every weak solution $u(\cdot, \tau ; \psi)$ of (3) with initial data $\psi \in B$, a bounded set of $\mathcal{H}$, satisfies the inequality

$$
\|u(t)\|^{2} \leq\|u(r)\|^{2}+C \int_{r}^{t}\left(c_{7}(s)+1\right) d s, \text { for all } \tau \leq r \leq t \leq T, T>\tau,
$$

where $c_{7}(t)=\sum_{i \in\{1,3,4,6\}} c_{i}(t)$ and $C=C(T, B)>0$.

Proof. Arguing as in (19), using (6) and that by Lemma $8\|u(t)\| \leq\left\|u_{t}\right\|_{C_{h}} \leq$ $C=C(T, B)$, for $t \geq \tau$, we obtain

$$
\begin{aligned}
\frac{d\|u\|^{2}}{d t} & +\lambda_{1}\|u\|^{2}+\|u\|_{1}^{2}+2 \eta|u|_{p}^{p} \leq 2\left\langle F_{2}\left(t, u_{t}\right), u\right\rangle+2 c_{1}(t)+2 C\left(c_{3}(t)+C c_{4}(t)\right) \\
& \leq 2\left\|F_{2}\left(t, u_{t}\right)\right\|_{-1}^{2}+\frac{1}{2}\|u\|_{1}^{2}+2 c_{1}(t)+2 C\left(c_{3}(t)+C c_{4}(t)\right) .
\end{aligned}
$$


By Gronwall's lemma, for $\tau \leq r \leq t$ we have

$$
\begin{aligned}
\| u(t) & \left\|^{2}+\frac{1}{2} \int_{r}^{t} e^{-\lambda_{1}(t-s)}\right\| u(s) \|_{1}^{2} d s \\
\leq & e^{-\lambda_{1}(t-r)}\|u(r)\|^{2}+2 \int_{r}^{t} e^{-\lambda_{1}(t-s)}\left\|F_{2}\left(s, u_{s}\right)\right\|_{-1}^{2} d s \\
& +2 \int_{r}^{t} e^{-\lambda_{1}(t-s)}\left(c_{1}(s)+C\left(c_{3}(s)+C c_{4}(s)\right)\right) d s .
\end{aligned}
$$

Note that from (8) it follows

$$
\begin{aligned}
2 \int_{r}^{t} e^{-\lambda_{1}(t-s)}\left\|F_{2}\left(s, u_{s}\right)\right\|_{-1}^{2} d s \leq & \int_{r}^{t} e^{-\lambda_{1}(t-s)} c_{6}(s) d s \\
& +K \int_{r}^{t} e^{-\lambda_{1}(t-s)} \int_{-\infty}^{0} e^{\lambda_{1} p}\left\|u_{s}(p)\right\|_{1}^{2} d p d s \\
\leq & \int_{r}^{t} e^{-\lambda_{1}(t-s)} c_{6}(s) d s \\
& +K(t-r) \int_{-\infty}^{t} e^{-\lambda_{1}(t-p)}\|u(p)\|_{1}^{2} d p
\end{aligned}
$$

for $\tau \leq r \leq t$. Therefore, as from (18) we have $\int_{-\infty}^{t} e^{-\lambda_{1}(t-p)}\|u(p)\|_{1}^{2} d s \leq C$, it yields

$$
\begin{aligned}
\| u(t) & \left\|^{2}+\frac{1}{2} \int_{r}^{t} e^{-\lambda_{1}(t-s)}\right\| u(s) \|_{1}^{2} d s \\
\leq & e^{-\lambda_{1}(t-r)}\|u(r)\|^{2}+\int_{r}^{t} e^{-\lambda_{1}(t-s)} c_{6}(s) d s \\
& +K C(t-r)+2 \int_{r}^{t} e^{-\lambda_{1}(t-s)}\left(c_{1}(s)+C\left(c_{3}(s)+C c_{4}(s)\right)\right) d s \\
& \leq\|u(r)\|^{2}+C \int_{r}^{t}\left(\sum_{i \in\{1,3,4,6\}} c_{i}(s)+1\right) d s .
\end{aligned}
$$

The proof is then complete.

We also need the following technical result.

Lemma 11 Let $t \mapsto J_{n}(t), t \mapsto J(t), t \in[\tau, T]$, be continuous non-increasing functions such that $J_{n}(t) \rightarrow J(t)$ for a.a. $t$ as $n \rightarrow \infty$. Then for all $t_{0} \in(\tau, T]$ and any sequence $t_{n} \rightarrow t_{0}$ we have

$$
\lim \sup _{n \rightarrow \infty} J_{n}\left(t_{n}\right) \leq J\left(t_{0}\right)
$$

If, moreover, $J_{n}(\tau) \rightarrow J(\tau)$, then the result is true also for $t_{0}=\tau$.

Proof. Take $t_{0} \in(\tau, T]$. Let $\tau<t_{m}<t_{0}$ be such that $J_{n}\left(t_{m}\right) \rightarrow J\left(t_{m}\right)$ for every $m \in \mathbb{N}$ and $t_{m} \rightarrow t_{0}$. We can assume that $t_{m}<t_{n}$. Since $J_{n}$ are 
non-increasing, we obtain

$$
J_{n}\left(t_{n}\right)-J\left(t_{0}\right) \leq\left|J_{n}\left(t_{m}\right)-J\left(t_{m}\right)\right|+\left|J\left(t_{m}\right)-J\left(t_{0}\right)\right| .
$$

Thus for any $\varepsilon>0$ there exist $t_{m}$ and $n_{0}\left(t_{m}\right)$ such that $J_{n}\left(t_{n}\right)-J\left(t_{0}\right) \leq \varepsilon$, for all $n \geq n_{0}$, and the result follows.

The last result follows in the same way by using $t_{m}=\tau$.

As we have mentioned, all the estimates obtained in Lemmas 8 and 10, and Corollary 9 are also true for the Galerkin approximation introduced in (15). This allows to conclude that these solutions $u^{n}$ exist globally on every interval $[\tau, T]$. In addition, we note that the bounds for $u^{n}$ are uniformly in $n \in \mathbb{N}$. Also, we have:

Lemma 12 Assuming conditions (5)-(8), the sequence

$$
\left(\frac{d u^{n}}{d t}\right)_{n \in \mathbb{N}}
$$

is bounded in $L^{q}\left(\tau, T ; H^{-r}(\mathcal{O})\right)$, for any $T>\tau$, where $r$ fulfills

$$
r \geq \max \left\{1, N\left(\frac{1}{q}-\frac{1}{2}\right)\right\} .
$$

Proof. By (5) and Corollary 9, the sequence $\left(G\left(\cdot, u_{.}^{n}\right)\right)_{n \in \mathbb{N}}$ is bounded in $L^{q}\left(\tau, T ; L^{q}(\mathcal{O})\right)$ and by (8) we obtain that $\left(F_{2}\left(\cdot, u_{.}^{n}\right)\right)_{n \in \mathbb{N}}$ is bounded in the space $L^{2}\left(\tau, T ; V^{\prime}\right)$. Also, condition (6) implies that $\left(F_{1}\left(\cdot, u_{.}^{n}\right)\right)_{n \in \mathbb{N}}$ is bounded in $L^{2}(\tau, T ; H)$, and then in $L^{2}\left(\tau, T ; V^{\prime}\right)$. Hence, the equality

$$
\frac{d u^{n(t)}}{d t}=P_{n}\left(-A u^{n}(t)+F_{2}\left(t, u_{t}^{n}\right)+F_{1}\left(t, u_{t}^{n}\right)-G\left(t, u^{n}(t)\right)\right)
$$

together with the fact that $\left\|P_{n} v\right\|_{-1} \leq\|v\|_{-1}$ (due to the choice of the special basis, see [29, Lemma 7.5] for the particular case of the Laplacian operator), imply that $\left(\frac{d u^{n}}{d t}\right)_{n \in \mathbb{N}}$ is bounded in $L^{2}\left(\tau, T ; V^{\prime}\right)+L^{q}\left(\tau, T ; L^{q}(\mathcal{O})\right)$.

From the Sobolev embedding theorem (see Lemma 4) we obtain that the embedding $L^{q}(\mathcal{O}) \subset H^{-r}(\mathcal{O})$ is continuous. Thus, $\left(\frac{d u^{n}}{d t}\right)_{n \in \mathbb{N}}$ is also bounded in $L^{q}\left(\tau, T ; H^{-r}(\mathcal{O})\right)$.

We now can conclude that there exists a subsequence of solutions of the Galerkin approximations, denoted also by $\left(u^{n}\right)_{n \in \mathbb{N}}$, with $u^{n} \in L^{2}\left(-\infty,-h ; V_{n}\right) \times$ 
$C\left([-h, T] ; V_{n}\right)$, such that for some $u$

$$
\begin{aligned}
u^{n} & \rightarrow u \text { weakly star in } L^{\infty}(\tau, T ; H), \\
u^{n} & \rightarrow u \text { weakly in } L^{2}(-\infty, T ; V) \text { and } L^{p}\left(\tau, T ; L^{p}(\mathcal{O})\right), \\
\frac{d u^{n}}{d t} & \rightarrow \frac{d u}{d t} \text { weakly in } L^{q}\left(\tau, T ; H^{-r}(\mathcal{O})\right),
\end{aligned}
$$

for every $T>\tau$. Also, a standard compactness theorem (see, e.g. Chapter 5.2 in [23]) implies that

$$
u^{n} \rightarrow u \text { strongly in } L^{2}(\tau, T ; H)
$$

To obtain the conclusion of Theorem 6 we show the following

Lemma 13 Under conditions (5)-(10), the limit point u given in (22) and (23) is a weak solution of (3).

Proof. Due to the choice of the special basis of eigenfunctions, by the properties of the projections $P_{n}$ it is easily seen that $\left(P_{n} \psi(\cdot)\right)_{n \in \mathbb{N}}$ tends to $\psi$ in $\mathcal{H}$. Indeed, it is easy to see that $P_{n} \psi \rightarrow \psi$ in $C([-h, 0], H)$ and, since by the choice of the basis we have $\left\|P_{n} u\right\|_{V} \leq\|u\|_{V}[29$, Lemma 7.5], for any $\varepsilon>0$ one can find $T(\varepsilon)<0$ and $N(\varepsilon, T)$ such that

$$
\begin{gathered}
\int_{-\infty}^{T}\left\|P_{n} \psi(s)\right\|_{V}^{2} d s \leq \int_{-\infty}^{T}\|\psi(s)\|_{V}^{2} d s \leq \varepsilon, \\
\int_{T}^{0}\left\|P_{n} \psi(s)-\psi(s)\right\|_{V}^{2} d s \leq \varepsilon, \text { if } n \geq N,
\end{gathered}
$$

so $P_{n} \psi \rightarrow \psi$ in $L^{2}(-\infty, 0 ; V)$.

By conditions (9)-(10) we have straightforwardly $G\left(\cdot, u^{n}(\cdot)\right) \rightarrow G(\cdot, u(\cdot))$ weakly in $L^{q}\left(\tau, T ; L^{q}(\mathcal{O})\right), F_{2}\left(\cdot, u_{.}^{n}\right) \rightarrow F_{2}(\cdot, u$. $)$ weakly in $L^{2}\left(\tau, T ; V^{\prime}\right)$.

On the other hand, condition (6) implies that $F_{1}\left(\cdot, u_{.}^{n}\right)$ is bounded in $L^{2}(\tau, T ; H)$, so that

$$
F_{1}\left(\cdot, u_{.}^{n}\right) \rightarrow \zeta_{h} \text { weakly in } L^{2}(\tau, T ; H) .
$$

Then passing to the limit we obtain that $u$ is a weak solution of the following equation

$$
\frac{d u}{d t}+A u=F_{2}\left(t, u_{t}\right)+\zeta_{h}-G(t, u), \quad \forall t \geq \tau .
$$

We have to show that $F_{1}(\cdot, u)=.\zeta_{h}(\cdot) \in C([\tau, T] ; H)$.

Firstly, let us prove that for any sequence $t_{n} \rightarrow t_{0}$ we have $u^{n}\left(t_{n}\right) \rightarrow u\left(t_{0}\right)$ weakly in $H$. The boundedness of $\left(u^{n}\left(t_{n}\right)\right)_{n \in \mathbb{N}}$ in $H$ implies the existence of a subsequence converging weakly in $H$ to some $\xi \in H$. If we check that then every subsequence contains a subsequence with limit point $u\left(t_{0}\right)$, then a 
standard argument would imply that the whole sequence converges weakly to $u\left(t_{0}\right)$, i.e. $\xi=u\left(t_{0}\right)$. Indeed, let $u^{n_{k}}\left(t_{n_{k}}\right) \rightarrow \xi$ weakly in $H$. Integrating in (15) we have that for any $e_{j}$ for $n_{k} \geq j$,

$$
\begin{aligned}
\left(u^{n_{k}}\left(t_{n_{k}}\right), e_{j}\right) & =\int_{\tau}^{t_{n_{k}}}\left(-A u^{n_{k}}(t)+F_{2}\left(t, u_{t}^{n_{k}}\right)+F_{1}\left(t, u_{t}^{n_{k}}\right)-G\left(t, u^{n_{k}}\right), e_{j}\right) d t \\
& +\left(u^{n_{k}}(\tau), e_{j}\right) \\
& \rightarrow\left(u(\tau), e_{j}\right)+\int_{\tau}^{t_{0}}\left(-A u(t)+F_{2}\left(t, u_{t}\right)+\zeta_{h}-G(t, u), e_{j}\right) d t
\end{aligned}
$$

as $n \rightarrow \infty$. Then

$$
\left(\xi, e_{j}\right)=\left(u(\tau), e_{j}\right)+\int_{\tau}^{t_{0}}\left(-A u(t)+F_{2}\left(t, u_{t}\right)+\zeta_{h}-G(t, u), e_{j}\right) d t .
$$

As the system $\left\{e_{j}\right\}_{j \geq 1}$ is dense in $V \cap L^{p}(\mathcal{O})$ we have

$$
\xi=\psi(0)+\int_{\tau}^{t_{0}}\left(-A u(t)+F_{2}\left(t, u_{t}\right)+\zeta_{h}-G(t, u)\right) d t \quad \text { in } V^{\prime}+L^{q}(\mathcal{O}) .
$$

But then equality (13) for $u$ (replacing $F_{1}$ by $\zeta_{h}$ ) implies that $\xi=u\left(t_{0}\right)$.

Next, let us check that $u^{n}\left(t_{n}\right) \rightarrow u\left(t_{0}\right)$ strongly in $H$ for any sequence $t_{n} \rightarrow$ $t_{0}, t_{n}, t_{0} \in[\tau, T]$. This would imply, as $u:[\tau, T] \rightarrow H$ is continuous, that $u^{n} \rightarrow u$ in $C([\tau, T] ; H)$. We know that $u^{n}\left(t_{n}\right) \rightarrow u\left(t_{0}\right)$ weakly in $H$. To see the strong convergence it is enough to prove that limsup $\left\|u^{n}\left(t_{n}\right)\right\| \leq\left\|u\left(t_{0}\right)\right\|$, because then $\lim \left\|u^{n}\left(t_{n}\right)\right\|=\left\|u\left(t_{0}\right)\right\|$, which gives $u^{n}\left(t_{n}\right) \rightarrow u\left(t_{0}\right)$ strongly in $H$.

Arguing as in Lemma 10 we can obtain the estimate (21) for the solutions of (15), which means that

$$
\left\|u^{n}(t)\right\|^{2} \leq\left\|u^{n}(r)\right\|^{2}+C \int_{r}^{t}\left(c_{7}(s)+1\right) d s, \quad \text { for } \tau \leq r \leq t \leq T .
$$

We can then define the functions

$$
J_{n}(t)=\left\|u^{n}(t)\right\|^{2}-C \int_{\tau}^{t}\left(c_{7}(s)+1\right) d s
$$

which are therefore non-decreasing and continuous. Notice that by (6) and (24) we have

$$
\int_{r}^{t}\left\|\zeta_{h}(s)\right\| d s \leq \liminf \int_{r}^{t}\left\|F_{1}\left(s, u_{s}^{n}\right)\right\| d s \leq \int_{r}^{t}\left(c_{3}(t)+c_{4}(t) C\right) d s,
$$

for $[r, t] \subset[\tau, T]$, since $\left\|u^{n}(t)\right\| \leq C$ for $t \in[\tau, T]$. Then, we can repeat the same lines of Lemma 10 obtaining that

$$
J(t)=\|u(t)\|^{2}-C \int_{\tau}^{t}\left(c_{7}(s)+1\right) d s
$$


is also a continuous and non-decreasing function. From (23) we obtain that $J_{n}(t) \rightarrow J(t)$ for a.a. $t \in[\tau, T]$ and it is clear that $J_{n}(\tau) \rightarrow J(\tau)$, as $n \rightarrow \infty$. Then by Lemma 11 we have $\lim \sup J_{n}\left(t_{n}\right) \leq J\left(t_{0}\right)$ and then $\lim \sup \left\|u^{n}\left(t_{n}\right)\right\| \leq$ $\left\|u\left(t_{0}\right)\right\|$, as $n \rightarrow \infty$.

Finally, $u^{n} \rightarrow u$ in $C([\tau, T] ; H)$ implies by $(6)$ that $\zeta_{h}=F_{1}(\cdot, u$. $)$. Hence $u$ is a solution of (3).

\section{Existence of the pullback attractor}

In this section we define a multivalued non-autonomous dynamical system generated by the solutions of (3) and prove the existence of a global pullback attractor for it. We observe here that every weak solution of (3) can be extended to a globally defined one (i.e. for all $t \geq \tau, \tau \in \mathbb{R}$ ) by concatenating solutions.

Let $\mathcal{S}(\psi, \tau)$ be the set of all globally defined solutions $u(\cdot ; \tau, \psi)$ to (3) corresponding to initial data $\psi \in \mathcal{H}$ and $\tau \in \mathbb{R}$. We define the multivalued map $U: \mathbb{R}_{d} \times \mathcal{H} \rightarrow P(\mathcal{H})$ as follows

$$
U(t, \tau, \psi)=\left\{u_{t}: u(\cdot ; \tau, \psi) \in \mathcal{S}(\psi, \tau)\right\} \in \mathcal{H} .
$$

The next lemma can be proved in a similar way as in [6, Proposition 4] or [3, Lemma 5.1].

Lemma $14 U$ defined by (26) satisfies the strict process property $U(t, \tau, \psi)=$ $U(t, s, U(s, \tau, \psi))$ for all $\tau \leq s \leq t$ and $\psi \in \mathcal{H}$. Hence, $U$ is a strict $M N D S$.

Now we additionally assume the following condition: there exist $\sigma>0$ and $R_{0}>0$ such that

$$
\limsup _{\tau \rightarrow+\infty}\left|\int_{-\tau}^{0} c_{4}^{2}(s) d s-\sigma \tau\right| \leq R_{0}
$$

where the function $c_{4}$ has been introduced in (6). We assume also that

$$
\lambda_{1}-\frac{4 \sigma e^{\lambda_{1} h}}{\mu \eta}=: \lambda>0
$$

Note that if $p>2$, then (28) is satisfied by choosing $\mu>0$ large enough in (16). If $p=2$, then (28) is satisfied when $\sigma$ is small enough or $\eta$ is large enough.

Finally, we also assume that for $\lambda$ given by (28), we have

$$
\lim _{t \rightarrow-\infty} \int_{-\infty}^{t} e^{\lambda s} c(s) d s=0
$$


where the function $c$ has been defined in Lemma 8 .

Remark 15 Observe that a sufficient condition implying (29) is that

$$
\int_{-\infty}^{0} e^{\lambda s} c(s) d s<+\infty
$$

Indeed, thanks to the fact that

$$
\int_{-\infty}^{t} e^{\lambda s} c(s) d s=\int_{-\infty}^{0} e^{\lambda s} c(s) d s-\int_{t}^{0} e^{\lambda s} c(s) d s
$$

for any $t<0$, we can take now limits as $t$ goes to $-\infty$, and obtain the result.

For $R>0$, denote by $B_{\mathcal{H}}(0, R)$ the closed ball in $\mathcal{H}$ centered at 0 with radius $R$. In the sequel, let us consider the system $\mathcal{D}$ given by the multi-valued mappings $D: \mathbb{R} \rightarrow P(\mathcal{H})$ with $D(s) \subset B_{\mathcal{H}}(0, \varrho(s))$, which is supposed to satisfy

$$
\lim _{s \rightarrow-\infty} \varrho^{2}(s) e^{\lambda s}=0 .
$$

Of course, $\mathcal{D}$ satisfies the inclusion closed property (see Section 2).

Define

$$
S^{2}(t)=\frac{12 e^{\lambda_{1} h}}{1-d} \int_{-\infty}^{t} e^{-\lambda_{1}(t-r)+\int_{r}^{t} \frac{4 e^{\lambda_{1} h}}{\mu \eta} c_{4}^{2}(s) d s} c(r) d r
$$

and assume that

$$
S^{2}(0)=\frac{12 e^{\lambda_{1} h}}{1-d} \int_{-\infty}^{0} e^{\lambda_{1} r+\int_{r}^{0} \frac{4 e^{\lambda_{1} h}}{\mu \eta} c_{4}^{2}(s) d s} c(r) d r<\infty
$$

Then, for every $t \in \mathbb{R}$,

$$
S^{2}(t)=e^{-\lambda_{1} t-\int_{t}^{0} \frac{4 e^{\lambda_{1} h}}{\mu \eta} c_{4}^{2}(s) d s}\left(S^{2}(0)+\frac{12 e^{\lambda_{1} h}}{1-d} \int_{0}^{t} e^{\lambda_{1} r+\int_{r}^{0} \frac{4 e^{\lambda_{1} h}}{\mu \eta} c_{4}^{2}(s) d s} c(r) d r\right)<\infty
$$

because of $(30)$ and $c, c_{4}^{2} \in L_{l o c}^{1}(\mathbb{R})$.

Let us prove the existence of an absorbing set in the space $\mathcal{H}$.

Lemma 16 Assume conditions (5)-(10) and (27), (28), (29), (30). Then the family of balls $B=(B(s))_{s \in \mathbb{R}}, B(s)=B_{\mathcal{H}}(0, S(s))$ is pullback $\mathcal{D}$-absorbing in $\mathcal{H}$. In addition, $B \in \mathcal{D}$.

Proof. To see that $B$ is absorbing we have to prove that for every $D \in \mathcal{D}$ and 
for every $t \in \mathbb{R}$, there exists $T=T(t, D)$ such that

$$
\begin{aligned}
& \sup _{\substack{u_{t} \in U(t, t-\tau, \psi) \\
\psi \in D(t-\tau)}}\left\|u_{t}\right\|_{\mathcal{H}}^{2} \leq S^{2}(t), \\
&
\end{aligned}
$$

for $\tau \geq T$. We know from Lemma 8 that the left hand side of (31) can be estimated by

$k e^{-\lambda_{1}(\tau-h)+\int_{t-\tau}^{t} \frac{4 e^{\lambda_{1} h}}{\eta \mu} c_{4}^{2}(s) d s} \sup _{\psi \in D(t-\tau)}\|\psi\|_{\mathcal{H}}^{2}+\frac{6 e^{\lambda_{1} h}}{1-d} \int_{-\tau}^{0} e^{\lambda_{1} s+\int_{s}^{0} \frac{4 e^{\lambda_{1} h}}{\eta \mu} c_{4}^{2}(t+r) d r} c(t+s) d s$

for some appropriate positive constant $k>0$, and for any $\tau \geq 0$.

Clearly, the second term of the right hand side is bounded by $(1 / 2) S^{2}(t)$, for any $\tau \geq 0$, since making a change of variable we also can write

$$
S^{2}(t)=\frac{12 e^{\lambda_{1} h}}{1-d} \int_{-\infty}^{0} e^{\lambda_{1} s+\int_{s}^{0} \frac{4 e^{\lambda_{1} h}}{\mu \eta} c_{4}^{2}(t+r) d r} c(t+s) d s .
$$

On the other hand, thanks to (27), for big enough $\tau$ it holds

$$
e^{-\lambda_{1}(\tau-h)+\int_{t-\tau}^{t} \frac{4 e^{\lambda_{1} h}}{\eta \mu} c_{4}^{2}(s) d s} \leq e^{\lambda_{1} h+\frac{4 e^{\lambda_{1} h} \bar{R}_{0}}{\eta \mu}+\int_{0}^{t} \frac{4 e^{\lambda_{1} h}}{\eta \mu} c_{4}^{2}(s) d s} e^{-\lambda \tau},
$$

where $\bar{R}_{0}>R_{0}$, and therefore

$$
\begin{aligned}
& k e^{-\lambda_{1}(\tau-h)+\int_{t-\tau}^{t} \frac{4 e^{\lambda_{1} h}}{\eta \mu} c_{4}^{2}(t+s) d s} \sup _{\psi \in D(t-\tau)}\|\psi\|^{2} \\
& \quad \leq k e^{\lambda_{1} h+\frac{4 e^{\lambda_{1} h} \bar{R}_{0}}{\eta \mu}+\int_{0}^{t} \frac{4 e^{\lambda_{1} h}}{\eta \mu} c_{4}^{2}(s) d s} e^{-\lambda \tau} \sup _{\psi \in D(t-\tau)}\|\psi\|^{2} \rightarrow 0
\end{aligned}
$$

when $\tau \rightarrow \infty$, because $D \in \mathcal{D}$.

It remains to prove that $B \in \mathcal{D}$. By condition (27), for every $\varepsilon>0$ there exists $T_{\varepsilon}>0$ such that

$$
-\sigma \tau-R_{0}-\varepsilon \leq \int_{\tau}^{0} c_{4}^{2}(s) d s \leq-\sigma \tau+R_{0}+\varepsilon, \quad \text { for } \quad \tau \leq-T_{\varepsilon} .
$$

Then, for $r \leq \tau \leq-T_{\varepsilon}$ we obtain

$$
\begin{aligned}
\int_{r}^{\tau} c_{4}^{2}(s) d s & =\int_{r}^{0} c_{4}^{2}(s) d s-\int_{\tau}^{0} c_{4}^{2}(s) d s \\
& \leq-\sigma(r-\tau)+2\left(R_{0}+\varepsilon\right),
\end{aligned}
$$


and, consequently,

$$
\begin{aligned}
e^{\lambda \tau} S^{2}(\tau) & =\frac{12 e^{\lambda \tau} e^{\lambda_{1} h}}{1-d} \int_{-\infty}^{\tau} e^{-\lambda_{1}(\tau-r)+\int_{r}^{\tau} \frac{4 e^{\lambda_{1} h}}{\mu \eta} c_{4}^{2}(s) d s} c(r) d r \\
& \leq \frac{12 e^{\lambda \tau} e^{\lambda_{1} h}}{1-d} \int_{-\infty}^{\tau} e^{-\lambda_{1}(\tau-r)+\frac{4 e^{\lambda_{1} h}}{\mu \eta} \sigma(\tau-r)+\frac{8 e^{\lambda_{1} h}}{\mu \eta}\left(R_{0}+\varepsilon\right)} c(r) d r \\
& \leq \frac{12 e^{\lambda \tau} e^{\lambda_{1} h+\frac{8 e^{\lambda_{1} h}}{\mu \eta}\left(R_{0}+\varepsilon\right)}}{1-d} \int_{-\infty}^{\tau} e^{-\lambda(\tau-r)} c(r) d r \\
& \leq \frac{12 e^{\lambda_{1} h+\frac{8 e^{\lambda_{1} h}}{\mu \eta}\left(R_{0}+\varepsilon\right)}}{1-d} \int_{-\infty}^{\tau} e^{\lambda r} c(r) d r, \quad \text { for } \tau \leq T_{\varepsilon} .
\end{aligned}
$$

Finally, condition (29) implies that $B \in \mathcal{D}$.

After the next auxiliary lemma, we shall prove that the process $U$ given by (26) is pullback asymptotically compact.

Lemma 17 Assume the conditions of Lemma 16 and also (11) and (12).

(i) Let $\psi^{n} \in B$, where $B$ is bounded in $\mathcal{H}$, and $\psi^{n} \rightarrow \psi$ weakly in $L_{V}^{2}, \psi^{n}(0) \rightarrow$ $\psi(0)$ weakly in $H$. Then for any sequence $u^{n}\left(\cdot, \tau ; \psi^{n}\right)$ there exists a subsequence $u^{n_{k}}$ and a function $u$ such that $u^{n_{k}}$ converges to $u$ in $C([r, T] ; H)$ for all $\tau<r<T$. Moreover, $u^{n_{k}} \rightarrow u$ weakly in $L^{2}(\tau, T ; V)$ for all $T>\tau$.

(ii) If moreover $\psi^{n} \rightarrow \psi$ in $C_{h}$, then $u^{n_{k}} \rightarrow u$ in $C([\tau-h, T] ; H)$, for all $T>\tau$, and $u$ is a solution of (3) corresponding to the initial data $\psi$. In addition,

$$
\limsup _{n \rightarrow \infty}\left\|u_{T}^{n}-u_{T}\right\|_{L_{V}^{2}}^{2} \leq \frac{1}{1-b} e^{-\lambda_{1}(T-\tau)} \limsup _{n \rightarrow \infty}\left\|\psi^{n}-\psi\right\|_{L_{V}^{2}}^{2}
$$

so that if $\psi^{n} \rightarrow \psi$ in $L_{V}^{2}$, then $u_{T}^{n_{k}} \rightarrow u_{T}$ in $L_{V}^{2}$ for any $T>\tau$.

Remark 18 In statement (i) of the last lemma we note that, in particular, $u_{t}^{n_{k}} \rightarrow u_{t}$ in $C_{h}$ for all $t>h+\tau$, so that we have obtained a compactness property in the space $C_{h}$ for $t>h+\tau$. In addition, taking into account that

$$
\left\|u_{t}^{n_{k}}\right\|_{L_{V}^{2}}^{2}=\int_{\tau-t}^{0} e^{\lambda_{1} s}\left\|u_{t}^{n_{k}}(s)\right\|_{1}^{2} d s+\int_{-\infty}^{\tau-t} e^{\lambda_{1} s}\left\|u_{t}^{n_{k}}(s)\right\|_{1}^{2} d s
$$

we obtain that $u_{t}^{n_{k}}$ is bounded in $L_{V}^{2}$, and then one can prove that $u_{t}^{n_{k}} \rightarrow u_{t}$ weakly in $L_{V}^{2}$ for all $t \in[\tau, T]$, where $u_{t}(s)=\psi(s)$ for $s \leq 0$. Hence, $u^{n_{k}} \rightarrow u$ weakly in $L^{2}(-\infty, T ; V)$.

Proof. The proof follows the lines of the proof of Lemma 13. For the sake of completeness we write here the main steps.

In view of inequality (17) the sequence $u^{n}$ is bounded in $L^{\infty}(\tau, T ; H) \cap$ 
$L^{2}(\tau, T ; V)$, and $\left\|u_{t}^{n}\right\|_{C_{h}}$ is uniformly bounded in $[\tau, T]$. Further, by Lemma 9 and arguing as in the proof of Lemma 12 we obtain that $u^{n}$ is bounded in $L^{p}\left(\tau, T ; L^{p}(\mathcal{O})\right)$ and $F_{2}\left(\cdot, u_{.}^{n}\right), F_{1}\left(\cdot, u_{.}^{n}\right)$ and $G\left(\cdot, u^{n}(\cdot)\right)$ are bounded in $L^{2}\left(\tau, T ; V^{\prime}\right), L^{2}(\tau, T ; H)$ and $L^{q}\left(\tau, T ; L^{q}(\mathcal{O})\right)$, respectively. Hence, the equality

$$
\frac{d u^{n}}{d t}=-A u^{n}+F_{2}\left(t, u_{t}^{n}\right)+F_{1}\left(t, u_{t}^{n}\right)-G\left(t, u^{n}\right)
$$

implies that $\left(\frac{d u^{n}}{d t}\right)_{n \in \mathbb{N}}$ is bounded in $L^{2}\left(\tau, T ; V^{\prime}\right)+L^{q}\left(\tau, T ; L^{q}(\mathcal{O})\right)$. If we choose $r \geq \max \left\{1, N\left(\frac{1}{q}-\frac{1}{2}\right)\right\}$, then $\left(\frac{d u^{n}}{d t}\right)_{n \in \mathbb{N}}$ is bounded in $L^{q}\left(\tau, T ; H^{-r}(\mathcal{O})\right)$.

In the sequel, we will denote by $\left(u^{n}\right)_{n \in \mathbb{N}}$ a sequence and any of its subsequences. Notice that because of the previous boundedness, we also get the same convergences as in (22) and (23). Moreover,

$$
F_{1}\left(t, u_{t}^{n}\right) \rightarrow \zeta_{h} \text { weakly in } L^{2}(\tau, T ; H) .
$$

In view of conditions $(9)-(10)$, we have that $G\left(\cdot, u^{n}(\cdot)\right) \rightarrow G(\cdot, u(\cdot))$ weakly in $L^{q}\left(\tau, T ; L^{q}(\mathcal{O})\right), F_{2}\left(\cdot, u_{.}^{n}\right) \rightarrow F_{2}(\cdot, u$. $)$ weakly in $L^{2}\left(\tau, T ; V^{\prime}\right)$, see Remark 18.

Since $\left\|u^{n}(t)\right\|$ is uniformly bounded in $[\tau, T]$ and the embedding $H \subset H^{-r}(\mathcal{O})$ is compact, using the Ascoli-Arzelà theorem we can show that $u^{n} \rightarrow u$ in $C\left([\tau, T], H^{-r}(\mathcal{O})\right)$. Then a standard argument implies that $u^{n}\left(t_{n}\right) \rightarrow u\left(t_{0}\right)$ weakly in $H$ for any sequence $t_{n} \rightarrow t_{0}, t_{n}, t_{0} \in[\tau, T]$.

Now, we need to check that $u^{n}\left(t_{n}\right) \rightarrow u\left(t_{0}\right)$ strongly in $H$ for any sequence $\left(t_{n}\right)_{n \in \mathbb{N}}, t_{n}, t_{0} \in[r, T]$, for any $r \in[\tau, T]$. This would imply, as $u:[r, T] \rightarrow H$ is continuous, that $u^{n} \rightarrow u$ in $C([r, T], H)$. As mentioned in the proof of Lemma 13 for this it is enough to obtain

$$
\limsup _{n \rightarrow \infty}\left\|u^{n}\left(t_{n}\right)\right\| \leq\left\|u\left(t_{0}\right)\right\| .
$$

In view of Lemma 10 the continuous functions

$$
J_{n}(t)=\left\|u^{n}(t)\right\|^{2}-C \int_{\tau}^{t}\left(c_{7}(s)+1\right) d s
$$

are non-increasing in $[\tau, T]$. Passing to the limit we obtain that $u(\cdot)$ is a solution of the following problem:

$$
\begin{aligned}
\frac{d u}{d t}+A u & =F_{2}\left(t, u_{t}\right)+\zeta_{h}-G(t, u), \\
u_{t}(\tau) & =\psi(0), u_{0}=\psi \text { in } L_{V}^{2} .
\end{aligned}
$$

We note $F_{1}\left(s, u_{s}^{n}\right) \rightarrow \zeta_{h}$ weakly in $L^{2}(\tau, T ; H)$ implies that $\zeta_{h}$ satisfies (25). Then repeating the same calculations of Lemma 10 we obtain that the con- 
tinuous function

$$
J(t)=\|u(t)\|^{2}-C \int_{\tau}^{t}\left(c_{7}(s)+1\right) d s
$$

is also non-increasing in $[\tau, T]$. Moreover, (23) implies, passing to a subsequence, that $J_{n}(t) \rightarrow J(t)$ for a.a. $t \in(\tau, T)$. Therefore, by Lemma 11 we have (33).

Applying now a diagonal argument we prove that the result is valid in an arbitrary interval $\tau \leq r \leq T$.

Assume now that, in addition, $\psi^{n} \rightarrow \psi$ in $C_{h}$. Then arguing as before one can check that $u^{n} \rightarrow u$ in $C([\tau-h, T] ; H)$. Hence, it follows from (4) that $F_{1}\left(t, u_{t}\right)=\zeta_{h}$, and then $u$ is a solution of (3) corresponding to the initial data $\psi$.

Assume finally that $\psi^{n} \rightarrow \psi$ in $L_{V}^{2}$, and let us check that $u_{T}^{n} \rightarrow u_{T}$ in $L_{V}^{2}$ for any $T>\tau$. In order to prove that, we want to get the estimate (32). Indeed, the difference $v^{n}=u^{n}-u$ satisfies

$$
\begin{aligned}
\frac{d}{d t}\left\|v^{n}\right\|^{2}+\lambda_{1}\left\|v^{n}\right\|^{2}+\left\|v^{n}\right\|_{1}^{2} \leq & 2\left\|F_{2}\left(t, u_{t}^{n}\right)-F_{2}\left(t, u_{t}\right)\right\|_{-1}^{2}+\frac{1}{2}\left\|v^{n}\right\|_{1}^{2} \\
& +2\left(F_{1}\left(t, u_{n}^{t}\right)-F_{1}\left(t, u_{t}\right), v^{n}\right) \\
& -2\left\langle G\left(t, u^{n}\right)-G(t, u), v^{n}\right\rangle_{q} .
\end{aligned}
$$

Then Gronwall's lemma and (12) imply

$$
\begin{aligned}
& \left\|v^{n}(T)\right\|^{2}+\frac{1-b}{2} \int_{\tau}^{T} e^{-\lambda_{1}(T-s)}\left\|v^{n}(s)\right\|_{1}^{2} d s \\
& \leq e^{-\lambda_{1}(T-\tau)}\left\|v^{n}(\tau)\right\|^{2}+\frac{b}{2} \int_{-\infty}^{\tau} e^{-\lambda_{1}(T-s)}\left\|v^{n}(s)\right\|_{1}^{2} d s \\
& \quad+2 \int_{\tau}^{T} e^{-\lambda_{1}(T-s)}\left(F_{1}\left(s, u_{s}^{n}\right)-F_{1}\left(s, u_{s}\right), v^{n}(s)\right) d s \\
& \quad-2 \int_{\tau}^{T} e^{-\lambda_{1}(T-s)}\left\langle G\left(s, u^{n}(s)\right)-G(s, u(s)), v^{n}(s)\right\rangle_{q} d s .
\end{aligned}
$$

By $\psi^{n}(0) \rightarrow \psi(0)$ in $H$ we obtain that

$$
\lim _{n \rightarrow \infty} e^{-\lambda_{1}(T-t)}\left\|v^{n}(\tau)\right\|^{2}=0
$$

and by (22) and (23),

$$
\lim _{n \rightarrow \infty} \int_{\tau}^{T} e^{-\lambda_{1}(T-s)}\left(F_{1}\left(s, u_{s}^{n}\right)-F_{1}\left(s, u_{s}\right), v^{n}(s)\right) d s=0,
$$

and

$$
\lim _{n \rightarrow \infty} \int_{\tau}^{T} e^{-\lambda_{1}(T-s)}\left\langle G(s, u(s)), v^{n}(s)\right\rangle_{q} d s=0
$$


Finally, by (9) and (11) we have

$$
\begin{aligned}
& \limsup _{n \rightarrow \infty} \int_{\tau}^{T} e^{-\lambda_{1}(T-s)}\left\langle-G\left(s, u^{n}(s)\right), u^{n}(s)-u(s)\right\rangle_{q} d s \\
& =\int_{\tau}^{T} e^{-\lambda_{1}(T-s)}\langle G(s, u(s)), u(s)\rangle_{q} d s \\
& \quad-\liminf _{n \rightarrow \infty} \int_{\tau}^{T} e^{-\lambda_{1}(T-s)}\left\langle G\left(s, u^{n}(s)\right), u^{n}(s)\right\rangle_{q} d s \leq 0 .
\end{aligned}
$$

Then, from (34), we get

$$
\begin{aligned}
& \limsup _{n \rightarrow \infty} \int_{\tau}^{T} e^{-\lambda_{1}(T-s)}\left\|v^{n}(s)\right\|_{1}^{2} d s \\
& \quad \leq \frac{b}{1-b} \limsup _{n \rightarrow \infty} \int_{-\infty}^{\tau} e^{-\lambda_{1}(T-s)}\left\|v^{n}(s)\right\|_{1}^{2} d s \\
& \quad=\frac{b}{1-b} e^{-\lambda_{1}(T-\tau)} \limsup _{n \rightarrow \infty} \int_{-\infty}^{0} e^{\lambda_{1} r}\left\|\psi^{n}(r)-\psi(r)\right\|_{1}^{2} d r
\end{aligned}
$$

and, because of

$$
\begin{aligned}
\left\|v_{T}^{n}\right\|_{L_{V}^{2}}^{2} & =\int_{\tau-T}^{0} e^{\lambda_{1} s}\left\|v_{T}^{n}(s)\right\|_{1}^{2} d s+\int_{-\infty}^{\tau-T} e^{\lambda_{1} s}\left\|v_{T}^{n}(s)\right\|_{1}^{2} d s \\
& =\int_{\tau}^{T} e^{-\lambda_{1}(T-s)}\left\|v^{n}(s)\right\|_{1}^{2} d s+e^{-\lambda_{1}(T-\tau)} \int_{-\infty}^{0} e^{\lambda_{1} r}\left\|v^{n}(r+\tau)\right\|_{1}^{2} d r
\end{aligned}
$$

then we have

$$
\limsup _{n \rightarrow \infty}\left\|v_{T}^{n}\right\|_{L_{V}^{2}}^{2} \leq \frac{1}{1-b} e^{-\lambda_{1}(T-\tau)} \limsup _{n \rightarrow \infty} \int_{-\infty}^{0} e^{\lambda_{1} r}\left\|\psi^{n}(r)-\psi(r)\right\|_{1}^{2} d r=0,
$$

and therefore the result is completely proved.

Corollary 1 The map $U$ has compact values.

Now we are ready to prove the asymptotic compactness.

Lemma 19 Assume the conditions of Lemma 16 and also (11) and (12). Then the MNDS $U$ is pullback $\mathcal{D}$-asymptotically compact.

Proof. Let $y_{n} \in U\left(t, t-\tau_{n}, D\left(t-\tau_{n}\right)\right)$, where $D \in \mathcal{D}$ and $\tau_{n} \rightarrow+\infty$. Then we have to prove that the sequence $y_{n}$ is pre-compact in $\mathcal{H}$. Let us first choose a large enough $T>0$ such that $U(t, t-T, D(t-T)) \subset B(t)$, where $B$ is the absorbing family. Then, for this fixed $T$, there exists $\bar{t}(D, t-T)>0$ such that for all $\tau_{n} \geq T+\bar{t}(D, t-T)$, we have

$U\left(t-T, t-\tau_{n}, D\left(t-\tau_{n}\right)\right)=U\left(t-T, t-T-\left(\tau_{n}-T\right), D\left(t-T-\left(\tau_{n}-T\right)\right)\right) \in B(t-T)$. 
On the other hand, we also have, for $\tau_{n} \geq T+\bar{t}(D, t-T)$,

$$
\begin{aligned}
U\left(t, t-\tau_{n}, D\left(t-\tau_{n}\right)\right) & =U\left(t, t-T, U\left(t-T, t-\tau_{n}, D\left(t-\tau_{n}\right)\right)\right) \\
& \subset U(t, t-T, B(t-T)) .
\end{aligned}
$$

Then $y_{n} \in U\left(t, t-T, \xi_{n}^{T}\right)$, where $\xi_{n}^{T} \in B(t-T)$. Let $u^{n}$ be a sequence of solutions such that $u_{t-T}^{n}=\xi_{n}^{T}$ and $u_{t}^{n}=y_{n}$. Observe that $y_{n}(\cdot-T)=\xi_{n}^{T}(\cdot)$ in $\mathcal{H}$. Also, it is clear that $u^{n}$ depends on $T$, but we omit this for simplicity of notation.

Since $B(t-T)$ is bounded in $\mathcal{H}$ we can assume (up to a subsequence) that $\xi_{n}^{T} \rightarrow \xi^{T}$ weakly in $L_{V}^{2}$. Also, since $y_{n} \in B(t)$ (for sufficiently large $n$ ), we have $y_{n} \rightarrow y$ weakly in $L_{V}^{2}$, and $y(\cdot-T)=\xi^{T}(\cdot)$ in $\mathcal{H}$.

In a similar way as in the proof of Lemma 17 it follows that $u^{n}$ converges to some function $u$ in the sense of (22), (23). Also, it is clear from the above convergences that $u(s)=y(s-t)$, for a.a. $s \leq t$, and then $u_{t}=y$ in $L^{2}(-\infty, 0 ; H)$. Lemma 17 implies, moreover, that

$$
u^{n} \rightarrow u \text { in } C([r, t], H), \text { for all } t-T<r<t .
$$

Hence, if we take $T>h$, then we obtain that $y_{n}=u_{t}^{n}$ converges to $y=u_{t}$ in $C_{h}$, so $u_{t}=y$ in $\mathcal{H}$.

Finally, we need to prove that $y_{n} \rightarrow y$ strongly in $L_{V}^{2}$. Thanks to (32), and taking into account that $\xi_{n}^{T}, \xi^{T} \in B(t-T)$, we get

$$
\begin{aligned}
\limsup _{n \rightarrow \infty}\left\|u_{t}^{n}-u_{t}\right\|_{L_{V}^{2}}^{2} & =\limsup _{n \rightarrow \infty}\left\|y_{n}-y\right\|_{L_{V}^{2}}^{2} \\
& \leq \frac{1}{1-b} e^{-\lambda_{1} T} \limsup _{n \rightarrow \infty}\left\|\xi_{n}^{T}-\xi^{T}\right\|_{L_{2}^{V}}^{2} \\
& \leq \frac{4}{1-b} e^{-\lambda_{1} T} S^{2}(t-T) .
\end{aligned}
$$

Notice that the right hand side of the last inequality can be made smaller than $1 / m$ for some $T=T_{m}$ because $\lambda_{1}>\lambda$ and $B \in \mathcal{D}$. Therefore, by a diagonal argument, we obtain a sequence $y_{n_{m}}$ converging strongly to $y$ in $L_{V}^{2}$ for $T \rightarrow+\infty$.

Lemma 20 Assume the conditions of Lemma 16 and also (11) and (12). Then the map $\psi \mapsto U(t, \tau, \psi)$ is upper semicontinuous for fixed $\tau \in \mathbb{R}, t \geq \tau$.

Proof. Assume the existence of $\psi \in \mathcal{H}$, of a neighborhood $\mathcal{U}$ of $U(t, \tau, \psi)$ and of a sequence $\xi^{n} \in U\left(t, \tau, \psi^{n}\right)$, where $\psi^{n} \rightarrow \psi$ in $\mathcal{H}$, such that $\xi^{n} \notin \mathcal{U}$. Lemma 17 implies that, up to a subsequence, $\xi^{n} \rightarrow \xi \in U(t, \tau, \psi)$ in $\mathcal{H}$. This is a contradiction. 
As a consequence of Lemmas 14, 16, 19, 20, Corollary 1 and Theorem 3 we have:

Theorem 21 Assume the conditions of Lemma 16 and (11) and (12). Then the MNDS generated by (3) possesses a pullback $\mathcal{D}$-attractor $\mathcal{A}$ in $\mathcal{H}$, which is strictly invariant.

\section{Application. Main result.}

Now we aim to analyze our motivating example (1). We will first state the assumptions on the functions appearing in the equation. Then we will check that all the assumptions established for the abstract equation are fulfilled in this particular case.

Let $p \geq 2$ and $q=\frac{p}{p-1}$. We consider a function $g: \mathcal{O} \times \mathbb{R} \times \mathbb{R} \rightarrow \mathbb{R}$, which is measurable with respect to $x \in \mathcal{O}$ and jointly continuous with respect to $(t, v) \in \mathbb{R}^{2}$, and such that

$$
\begin{aligned}
& g(x, t, v) v \geq \eta|v|^{p}-\delta_{1}(t), \\
& |g(x, t, v)|^{q} \leq \rho|v|^{p}+\delta_{2}(t),
\end{aligned}
$$

where $\eta, \rho$ are positive constants, and $\delta_{1}, \delta_{2}$ are positive functions which belong to $L_{\text {loc }}^{1}(\mathbb{R})$. Define $G: \mathbb{R} \times L^{p}(\mathcal{O}) \rightarrow L^{q}(\mathcal{O})$ as $G(t, v)(x):=g(x, t, v(x))$, for $v \in L^{p}(\mathcal{O})$ and $t \in \mathbb{R}, x \in \mathcal{O}$. Then

$$
\begin{aligned}
\langle G(t, v), v\rangle_{q} & =\int_{\mathcal{O}} g(x, t, v(x)) v(x) d x \\
& \geq \eta \int_{\mathcal{O}}|v(x)|^{p} d x-\delta_{1}(t) \int_{\mathcal{O}} d x \\
& =\eta|v|_{p}^{p} d x-\delta_{1}(t)|\mathcal{O}|,
\end{aligned}
$$

and

$$
\begin{aligned}
|G(t, v)|_{q}^{q} & =\int_{\mathcal{O}} g(x, t, v(x))^{q} d x \\
& \leq \delta_{2}(t) \int_{\mathcal{O}} d x+\rho \int_{\mathcal{O}}|v(x)|^{p} \\
& =\delta_{2}(t)|\mathcal{O}|+\rho|v|_{p}^{p},
\end{aligned}
$$

and therefore (5) holds.

Let us consider now condition (9). By $u^{n} \rightarrow u$ in $L^{2}(0, T ; H)$ we know that $u^{n}(t, x) \rightarrow u(t, x)$ for a.a. $(t, x) \in(\tau, T) \times \mathcal{O}$. Hence, the continuity of the map $v \mapsto g(x, t, v)$ implies that $g\left(x, t, u^{n}(t, x)\right) \rightarrow g(x, t, u(t, x))$ for a.a. $(t, x)$. 
Then (37) implies that

$$
\left\|G\left(\cdot, u^{n}(\cdot)\right)\right\|_{L^{q}\left(\tau, T ; L^{q}(\mathcal{O})\right)}^{q} \leq \int_{\tau}^{T}\left(\delta_{2}(t)|\mathcal{O}|+\rho\left|u^{n}(t)\right|_{p}^{p}\right) d t \leq C,
$$

and also that $G(\cdot, u(\cdot)) \in L^{q}\left(\tau, T ; L^{q}(\mathcal{O})\right)$. Hence, a standard lemma (see e.g. [23]) implies that $G\left(\cdot, u^{n}(\cdot)\right) \rightarrow G(\cdot, u(\cdot))$ weakly in $L^{q}\left(\tau, T ; L^{q}(\mathcal{O})\right)$. Therefore, (9) is satisfied.

We check now condition (11). It follows from (35) that

$$
g\left(x, t, u^{n}(t, x)\right) u^{n}(t, x) \geq-\delta_{1}(t),
$$

and then Lebesgue-Fatou's lemma (see [33]) implies

$$
\begin{aligned}
& \liminf _{n \rightarrow \infty} \int_{\tau}^{T} e^{-\lambda_{1}(T-s)}\left\langle G\left(t, u^{n}(s)\right), u^{n}(s)\right\rangle_{q} d s \\
& =\liminf _{n \rightarrow \infty}\left(\int_{\tau}^{T} \int_{\mathcal{O}} e^{-\lambda_{1}(T-s)} g\left(x, t, u^{n}(t, x)\right) u^{n}(t, x) d x d s\right) \\
& \geq \int_{\tau}^{T} \int_{\mathcal{O}} e^{-\lambda_{1}(T-s)} \liminf _{n \rightarrow \infty}\left(g\left(x, t, u^{n}(t, x)\right) u^{n}(t, x)\right) d x d s \\
& =\int_{\tau}^{T} \int_{\mathcal{O}} e^{-\lambda_{1}(T-s)} g(x, t, u(t, x)) u(t, x) d x d s \\
& =\int_{\tau}^{T} e^{-\lambda_{1}(T-s)}\langle G(t, u(s)), u(s)\rangle_{q} d s,
\end{aligned}
$$

so that (11) holds.

The map $(t, v) \mapsto G(t, v)$ is continuous, which follows from the continuity of $(t, v) \mapsto g(x, t, v)$, condition (35), the convergence $u^{n} \rightarrow u$ in $L^{2}(\tau, T ; H)$ and Lebesgue's theorem.

Let $f_{1}: \mathcal{O} \times \mathbb{R} \times \mathbb{R} \rightarrow \mathbb{R}$ be a continuous function such that

$$
\left|f_{1}(t, x, v)\right| \leq \delta_{3}(t)+\delta_{4}(t)|v|,
$$

where $\delta_{3}, \delta_{4}$ are positive functions such that $\delta_{3}^{2}, \delta_{4}^{2} \in L_{\text {loc }}^{1}(\mathbb{R})$. Thus $F_{1}: \mathbb{R} \times$ $C_{h} \rightarrow H$ given by

$$
F_{1}(t, \xi)(x):=f_{1}(x, t, \xi(-h, x)), \quad x \in \mathcal{O}, \xi \in C_{h},
$$

is such that

$$
\begin{aligned}
\left\|F_{1}(t, \xi)\right\|^{2} & =\int_{\mathcal{O}} \mid f_{1}\left(x, t,\left.\xi(-h, x)\right|^{2} d x\right. \\
& \leq 2 \int_{\mathcal{O}}\left(\delta_{3}^{2}(t)+\delta_{4}^{2}(t)\left|\xi^{2}(-h, x)\right|\right) d x \\
& =2 \delta_{3}^{2}(t)|\mathcal{O}|+2 \delta_{4}^{2}(t)\|\xi\|_{C_{h}}^{2},
\end{aligned}
$$


and therefore (6) is clearly satisfied.

Arguing as in the previous case, we obtain that the map $(t, \xi) \mapsto F_{1}(t, \xi)$ is continuous.

Define $F_{2}: \mathbb{R} \times L_{V}^{2} \rightarrow V^{\prime}$, as

$$
\begin{aligned}
\left\langle F_{2}(t, \psi), v\right\rangle & =-\int_{-\infty}^{t} \gamma(t-s)\langle\Delta \psi(s-t), v\rangle d s \\
& =\int_{\mathcal{O}}\left(\int_{-\infty}^{t} \gamma(t-s) \nabla \psi(x, s-t) d s\right) \nabla v(x) d x
\end{aligned}
$$

for $v \in V$, where the function $\gamma$ is the standard kernel defined as

$$
\gamma(\theta)=-\gamma_{0} e^{-d_{0} \theta}, \theta \geq 0,
$$

for some constants $\gamma_{0}>0$ and $d_{0}>\lambda_{1}$ such that

$$
\frac{4 \gamma_{0}^{2}}{d_{0}\left(d_{0}-\lambda_{1}\right)}<1
$$

which holds provided $d_{0}$ is large enough or $\gamma_{0}$ is sufficiently small. We observe that the condition $\gamma_{0}>0$ is not essential for the further calculations, but we keep it due to the physical motivation of the function $\gamma$ (see the Introduction).

Then,

$$
\begin{aligned}
& \left|\left\langle F_{2}(t, \psi), v\right\rangle\right| \\
& \leq \int_{-\infty}^{t}|\gamma(t-s)|\left(\int_{\mathcal{O}}|\nabla \psi(x, s-t)|^{2} d x\right)^{1 / 2}\left(\int_{\mathcal{O}}|\nabla v(x)|^{2} d x\right)^{1 / 2} d s \\
& =\int_{-\infty}^{t}|\gamma(t-s)|\|\psi(s-t)\|_{1}\|v\|_{1} d s, \quad \text { for } v \in V,
\end{aligned}
$$

and

$$
\begin{aligned}
\left\|F_{2}(t, \psi)\right\|_{-1} & =\sup _{\|v\|_{1} \leq 1}\left|\left\langle F_{2}(t, \psi), v\right\rangle\right| \\
& \leq \int_{-\infty}^{t} \gamma_{0} e^{-d_{0}(t-s)}\|\psi(s-t)\|_{1} d s \\
& =\int_{-\infty}^{0} \gamma_{0} e^{d_{0} s}\|\psi(s)\|_{1} d s \\
& \leq\left(\int_{-\infty}^{0} e^{\left(2 d_{0}-\lambda_{1}\right) s} \gamma_{0}^{2} d s\right)^{1 / 2}\|\psi\|_{L_{V}^{2}} \\
& =\left(\int_{0}^{\infty} e^{-\left(2 d_{0}-\lambda_{1}\right) s} \gamma_{0}^{2} d s\right)^{1 / 2}\|\psi\|_{L_{V}^{2}} \\
& \leq \frac{\gamma_{0}}{\left(2 d_{0}-\lambda_{1}\right)^{1 / 2}}\|\psi\|_{L_{V}^{2}}=: K^{1 / 2}\|\psi\|_{L_{V}^{2}}
\end{aligned}
$$


where we have used $d_{0}>\lambda_{1}$. Therefore,

$$
2\left\|F_{2}(t, \psi)\right\|_{-1}^{2} \leq 2 K\|\psi\|_{L_{V}^{2}}^{2}, \text { for } \psi \in L_{V}^{2},
$$

so (8) holds.

In addition, from (39),

$$
\begin{aligned}
2 \int_{\tau}^{t} e^{\lambda_{1} s} & \left\|F_{2}\left(s, u_{s}\right)\right\|_{-1}^{2} d s \leq 2 \int_{\tau}^{t} e^{\lambda_{1} s}\left(\int_{-\infty}^{s} \gamma_{0} e^{-d_{0}(s-r)}\|u(r)\|_{1} d r\right)^{2} d s \\
\leq & 2 \gamma_{0}^{2} \int_{\tau}^{t} e^{\lambda_{1} s}\left(\int_{-\infty}^{s} e^{-d_{0}(s-r)} d r\right)\left(\int_{-\infty}^{s} e^{-d_{0}(s-r)}\|u(r)\|_{1}^{2} d r\right) d s \\
\leq & \frac{2 \gamma_{0}^{2}}{d_{0}} \int_{\tau}^{t} e^{\lambda_{1} s}\left(\int_{-\infty}^{s} e^{-d_{0}(s-r)}\|u(r)\|_{1}^{2} d r\right) d s \\
= & \frac{2 \gamma_{0}^{2}}{d_{0}}\left[\int_{-\infty}^{\tau}\left(\int_{\tau}^{t} e^{\lambda_{1} s} e^{-d_{0}(s-r)}\|u(r)\|_{1}^{2} d s\right) d r\right. \\
& \left.+\int_{\tau}^{t}\left(\int_{r}^{t} e^{\lambda_{1} s} e^{-d_{0}(s-r)}\|u(r)\|_{1}^{2} d s\right) d r\right] \\
= & \frac{2 \gamma_{0}^{2}}{d_{0}}\left[\int_{-\infty}^{\tau} e^{d_{0} r}\|u(r)\|_{1}^{2}\left(\int_{\tau}^{t} e^{\left(\lambda_{1}-d_{0}\right) s} d s\right) d r\right. \\
& \left.+\int_{\tau}^{t} e^{d_{0} r}\|u(r)\|_{1}^{2}\left(\int_{r}^{t} e^{\left(\lambda_{1}-d_{0}\right) s} d s\right) d r\right] \\
\leq & \frac{2 \gamma_{0}^{2}}{d_{0}\left(d_{0}-\lambda_{1}\right)}\left[\int_{-\infty}^{\tau} e^{d_{0} r} e^{\left(\lambda_{1}-d_{0}\right) \tau}\|u(r)\|_{1}^{2} d r+\int_{\tau}^{t} e^{d_{0} r} e^{\left(\lambda_{1}-d_{0}\right) r}\|u(r)\|_{1}^{2} d r\right] \\
\leq & \frac{2 \gamma_{0}^{2}}{d_{0}\left(d_{0}-\lambda_{1}\right)}\left[\int_{-\infty}^{\tau} e^{d_{0} r} e^{\left(\lambda_{1}-d_{0}\right) r}\|u(r)\|_{1}^{2} d r+\int_{\tau}^{t} e^{\lambda_{1} r}\|u(r)\|_{1}^{2} d r\right] \\
\leq & \frac{2 \gamma_{0}^{2}}{d_{0}\left(d_{0}-\lambda_{1}\right)} \int_{-\infty}^{t} e^{\lambda_{1} r}\|u(r)\|_{1}^{2} d r
\end{aligned}
$$

and thus calling

$$
\frac{d}{2}:=\frac{2 \gamma_{0}^{2}}{d_{0}\left(d_{0}-\lambda_{1}\right)}
$$

condition (7) holds in view of (41).

In addition, it is clear from the above estimates that considering $u, v \in$ $L^{2}(-\infty, T ; V)$, for $t>\tau$ we have that

$$
2 \int_{\tau}^{t} e^{\lambda_{1} s}\left\|F_{2}\left(s, u_{s}\right)-F_{2}\left(s, v_{s}\right)\right\|_{-1}^{2} d s \leq \frac{2 \gamma_{0}^{2}}{d_{0}\left(d_{0}-\lambda_{1}\right)} \int_{-\infty}^{t} e^{\lambda_{1} r}\|u(r)-v(r)\|_{1}^{2} d r
$$

and thus (12) also holds taking $b=d$. 
The continuity of $(t, \psi) \mapsto F_{2}(t, \psi)$ follows from

$$
\begin{aligned}
\left\|F_{2}\left(t, \psi_{2}\right)-F_{2}\left(t, \psi_{1}\right)\right\|_{-1} & \leq \int_{-\infty}^{t} \gamma_{0} e^{-d_{0}(t-s)}\left\|\psi_{2}(s-t)-\psi_{1}(s-t)\right\|_{1} d s \\
& =\gamma_{0} \int_{-\infty}^{0} e^{d_{0} r}\left\|\psi_{2}(r)-\psi_{1}(r)\right\|_{1} d r \\
& \leq \gamma_{0}\left(\int_{0}^{\infty} e^{-\left(2 d_{0}-\lambda_{1}\right) r} d r\right)^{1 / 2}\left\|\psi_{2}-\psi_{1}\right\|_{L_{V}^{2}} \\
& =\frac{\gamma_{0}}{\left(2 d_{0}-\lambda_{1}\right)^{1 / 2}}\left\|\psi_{2}-\psi_{1}\right\|_{L_{V}^{2}}
\end{aligned}
$$

For condition (10) we note that for any $\psi \in L^{2}(\tau, T ; V)$,

$$
\begin{aligned}
\int_{\tau}^{T}\left\langle F_{2}\left(s, u_{s}^{n}\right), \psi(s)\right\rangle d s & =-\gamma_{0} \int_{\tau}^{T} \int_{-\infty}^{s} e^{-d_{0}(s-r)}\left\langle\Delta u^{n}(r) d r, \psi(s)\right\rangle d r d s \\
& =-\gamma_{0} \int_{\tau}^{T} \int_{-\infty}^{0} e^{\lambda_{1} r}\left\langle\Delta u^{n}(r+s), e^{\left(d_{0}-\lambda_{1}\right) r} \psi(s)\right\rangle d r d s
\end{aligned}
$$

For a.a. $s \in(0, T)$ we have $e^{\left(d_{0}-\lambda_{1}\right) \cdot} \psi(s) \in L_{V}^{2}$ and then $u^{n}(\cdot+s) \rightarrow u(\cdot+s)$ weakly in $L_{V}^{2}$ implies

$\int_{-\infty}^{0} e^{\lambda_{1} r}\left\langle\Delta u^{n}(r+s), e^{\left(d_{0}-\lambda_{1}\right) r} \psi(s)\right\rangle d r \rightarrow \int_{-\infty}^{0} e^{\lambda_{1} r}\left\langle\Delta u(r+s), e^{\left(d_{0}-\lambda_{1}\right) r} \psi(s)\right\rangle d r$.

Also, by (42) and the boundedness of $u^{n}$ in $L^{2}(-\infty, T ; V)$ we have

$$
\begin{aligned}
\left|\left\langle F_{2}\left(s, u_{s}^{n}\right), \psi(s)\right\rangle\right| & \leq \gamma_{0} \int_{-\infty}^{s} e^{-d_{0}(s-r)}\left\|u^{n}(r)\right\|_{1}\|\psi(s)\|_{1} d r \\
& =\gamma_{0} \int_{-\infty}^{0} e^{d_{0} r}\left\|u^{n}(r+s)\right\|_{1} d r\|\psi(s)\|_{1} \\
& \leq \gamma_{0}\left(\int_{0}^{\infty} e^{-\left(2 d_{0}-\lambda_{1}\right) r} d r\right)\left(\int_{-\infty}^{0} e^{\lambda_{1} r}\left\|u^{n}(r+s)\right\|_{1}^{2} d r\right)^{1 / 2}\|\psi(s)\|_{1} \\
& \leq \frac{\gamma_{0}}{\left(2 d_{0}-\lambda_{1}\right)^{1 / 2}} C\|\psi(s)\|_{1} .
\end{aligned}
$$

By Lebesgue's theorem we obtain that

$$
\int_{\tau}^{T}\left\langle F_{2}\left(s, u_{s}^{n}\right), \psi(s)\right\rangle d s \rightarrow \int_{\tau}^{T}\left\langle F_{2}\left(s, u_{s}\right), \psi(s)\right\rangle d s
$$

so that (10) holds.

Finally, as all conditions in Theorem 21 are satisfied we can reformulate the Cauchy problem for (1) with Dirichlet boundary conditions in the space $\mathcal{H}$ in the abstract form (3) and obtain the main result of the paper. 
Theorem 2 Assume conditions (35), (38), (41). Then the MNDS generated by (1) with Dirichlet boundary conditions possesses a pullback $\mathcal{D}$-attractor $\mathcal{A}$ in the space $\mathcal{H}$, which is strictly invariant.

Remark 22 It is not difficult to see that, considering a general continuous function $\gamma$, we could obtain the assumptions for the corresponding operator $F_{2}$ just assuming that

$$
\max \left\{\int_{0}^{\infty} e^{\lambda_{1} s}|\gamma(s)| d s, \int_{0}^{\infty} e^{\lambda_{1} s} \gamma^{2}(s) d s\right\}<\infty
$$

and

$$
4\left(\int_{0}^{\infty}|\gamma(r)| d r\right)\left(\int_{0}^{\infty} e^{\lambda_{1} r}|\gamma(r)| d r\right)<1 .
$$

\section{References}

[1] L. Arnold, "Random Dynamical Systems", Springer Monographs in Mathematics, Springer-Verlag, Berlin, 1998.

[2] T. Caraballo, I.D. Chueshov, J. Real, Pullback attractors for stochastic heat equations in materials with memory, Discrete Cont. Dyn. Systems, Series B, $\mathbf{9}$ (2008), 525-539.

[3] T. Caraballo, M.J. Garrido-Atienza, B. Schmalfuß, J. Valero, Non-autonomous and random attractors for delay random semilinear equations without uniqueness, Discrete Contin. Dyn. Syst., 21 (2008), 415-443.

[4] T. Caraballo, M.J. Garrido-Atienza, B. Schmalfuß, J. Valero, Asymptotic behaviour of a stochastic semilinear dissipative functional equation without uniqueness of solutions, Discrete Cont. Dyn. Systems, Series B (to appear) .

[5] T. Caraballo, P.E. Kloeden, Non-autonomous attractors for integro-differential evolution equations, Discrete Contin. Dyn. Syst., Series S, 2 (2009), 17-36.

[6] T. Caraballo, J. A. Langa, J. Valero, Global attractors for multivalued random dynamical systems, Nonlinear Anal., 48 (2002), 805-829.

[7] V.V. Chepyzhov, S. Gatti, M. Grasselli, A. Miranville, V. Patta, Trajectory and global attractors for evolutions equations with memory, Applied Mathematics Letters, 19 (2006), 87-96.

[8] V.V. Chepyzhov, E. Mainini, V. Pata, Stability of abstract linear semigroups arising from heat conduction with memory, Asymptotic Analysis, 50(3-4) (2006), 269-291.

[9] V.V. Chepyzhov, A. Miranville, On trajectory and global attractors for semilinear heat equations with fading memory, Indiana University Mathematics Journal, 55(1) (2006), 119-167. 
[10] V.V. Chepyzhov, M.I.Vishik, Trajectory attractors for reaction-diffusion systems, Topol. Methods Nonlinear Anal., 7 (1996), 49-76.

[11] V.V. Chepyzhov, M.I.Vishik, "Attractors for equations of mathematical physics", American Mathematical Society, Providence, Rhode Island, 2002.

[12] I. Chueshov, M. Scheutzow, Inertial manifolds and forms for stochastically perturbed retarded semilinear parabolic equations, J. Dynamics Differential Equations, 13 (2001), 355-380.

[13] B. D. Coleman and M. E. Gurtin, Equipresence and constitutive equations for rigid heat conduction, Z. Angew. Math. Phys., 18 (1967), 199-208.

[14] G. Da Prato, J. Zabczyk, "Stochastic equations in infinite dimensions", Cambridge University Press, Cambridge, 1992.

[15] M. Fabrizio and A. Morro, "Mathematical Problems in Linear Viscoelasticity," SIAM Studies in Applied Mathematics 12, SIAM, Philadelphia, 1992.

[16] S. Gatti, M. Grasselli, V. Pata, Lyapunov functionals for reaction-diffusion equations with memory, Mathematical Methods in the Applied Sciences, 28(14) (2005), 1725-1735.

[17] M. E. Gurtin and A. C. Pipkin, A general theory of heat conduction with finite wave speed, Arch. Rational Mech. Anal., 31 (1968) 113-126.

[18] Y. Hino, S. Murakami, T. Naito, "Functional Differential Equations with Infinite Delay", Lecture Notes in Mathematics, 1473, Springer-Verlag, Berlin, 1991.

[19] S. Hu, N. S. Papageorgiou, "Handbook of Multivalued Analysis", Vol. I, volume 419 of Mathematics and its Applications, Kluwer Academic Publishers, Dordrecht, 1997.

[20] A.V. Kapustyan, J. Valero, On the connectedness and asymptotic behavior of solutions of reaction-diffusion systems, J. Math. Anal. Appl., 323 (2006), 614633.

[21] A.V. Kapustyan, J. Valero, On the Kneser property for the complex GinzburgLandau equation and the Lotka-Volterra system with diffusion, J. Math. Anal. Appl., 357 (2009), 254-272.

[22] H. Kunita, "Stochastic flows and stochastic differential equations", Cambridge University Press, Cambridge, 1990.

[23] J.L. Lions, "Quelques méthodes de résolution des problèmes aux limites non linéaires", Gauthier-Villar, Paris, 1969.

[24] J.L. Lions, E.Magenes, "Problèmes aux limites non-homogènes et applications", Dunod, Paris, 1968.

[25] F. Morillas, J. Valero, Attractors for reaction-diffusion equations in $\mathbb{R}^{N}$ with continuous nonlinearity, Asympt. Anal., 44 (2005), 111-130. 
[26] F. Morillas, J. Valero, On the Kneser property for reaction-diffusion systems on unbounded domains, Topol. Appl., 156 (2009), 3029-3040.

[27] J. W. Nunziato, On heat conduction in materials with memory, Quart. Appl. Math., 29 (1971), 187-204.

[28] M. Renardy, W. J. Hrusa and J. A. Nohel, "Mathematical Problems in Viscoelasticity," Longman, Harlow; John Willey, New York, 1987.

[29] J. Robinson, "Infinite-Dimensional Dynamical Systems", Cambridge University Press, Cambridge, 2001.

[30] B. Schmalfuß, Attractors for the non-autonomous dynamical systems, In "International Conference on Differential Equations, Vol. 1, 2 (Berlin, 1999)", pp. 684-689, World Sci. Publishing, River Edge, NJ, 2000.

[31] S. Takeuchi, H. Asakawa and T. Yokota, Complex Ginzburg-Landau type equations with nonlinear Laplacian, in the "Proceedings of the international conference Nonlinear Partial Differential Equations and Their Applications (Shangai, 2003)", GAKUTO Internat. Ser. Math. Appl., 20 (2004), 315-332.

[32] M.I. Vishik, A.V. Fursikov, "Mathematical problems of statistical hydromechanics" Kluwer Academic Publishers, Dordrecht, 1988.

[33] K. Yosida, "Functional Analysis", Springer-Verlag, Berlin-Heidelberg, 1965.

[34] L. Wang, D. Xu, Asymptotic behavior of a class of reaction-diffusion equations with delays, J. Math. Anal. Appl., 281 (2003), 439-453. 